\title{
Systematic analysis of intrinsic enhancer-promoter compatibility in the mouse genome.
}

\author{
${ }^{1}$ Miguel Martinez-Ara, ${ }^{1,2}$ Federico Comoglio, ${ }^{1,3}$ Joris van Arensbergen, \\ ${ }^{1}$ Bas van Steensel ${ }^{*}$
}

${ }^{1}$ Division of Gene Regulation and Oncode Institute, Netherlands Cancer Institute, Amsterdam, the Netherlands

${ }^{2}$ Present address: enGene Statistics $\mathrm{GmbH}$, Basel, Switzerland

${ }^{3}$ Present address: Annogen B.V., Science park 406, Amsterdam, the Netherlands

*correspondence: b.v.steensel@nki.nl

\section{Abstract}

Gene expression is in part controlled by cis-regulatory elements (CREs) such as enhancers and repressive elements. Anecdotal evidence has indicated that a CRE and a promoter need to be biochemically compatible for promoter regulation to occur, but this compatibility has remained poorly characterised in mammalian cells. We used high-throughput combinatorial reporter assays to test thousands of CRE - promoter pairs from three Mb-sized genomic regions in mouse cells. This revealed that CREs vary substantially in their promoter compatibility, ranging from striking specificity for a single promoter to quantitative differences in activation across a broad set of promoters. More than half of the tested CREs exhibit significant promoter selectivity. Housekeeping promoters tend to have similar CRE preferences, but other promoters exhibit a wide diversity of compatibilities. Higher-order TF motif combinations may account for compatibility. CRE-promoter selectivity does not correlate with looping interactions in the native genomic context, suggesting that chromatin folding and compatibility are two orthogonal mechanisms that confer specificity to gene regulation.

\section{Keywords}

Enhancer, promoter, cis-regulatory element, repressor, massively parallel reporter assay, combinatorial, gene regulation, transcription. 
INTRODUCTION

How genes are regulated by cis-regulatory elements (CREs) such as enhancers and repressor elements is a long-standing topic in molecular biology [1-10]. One conundrum is how CREs 'choose' their target promoters. Some enhancers can activate multiple promoters in cis over short and long genomic distances [11-13], while others show remarkable specificity, regulating only one of its neighbouring promoters or even skipping one or more promoters to activate more distal ones. In part, 3D folding and compartmentalisation of the chromatin fibre help to establish this specificity, by facilitating certain enhancer-promoter contacts and curbing others [12-14].

However, there is also substantial evidence that biochemical (in)compatibility between CREs and promoters contributes to the specificity of their regulatory interactions. This is akin to a lock-and-key mechanism: proteins bound to the CRE and the promoter must be compatible in order to form a productive complex. Examples of such intrinsic selectivity have been documented particularly in Drosophila, and in some instances could be attributed to a specific sequence motif in the promoter [15-19]. Data obtained with massively parallel reporter assays (MPRAs) in Drosophila cells have suggested a general separation of enhancer-promoter compatibility into housekeeping and tissue-specific classes [20]. Some of this specificity may be determined by the recruitment of co-factors [21]. However, a thorough understanding of the underlying mechanisms is still lacking.

While several studies of individual enhancer-promoter combinations indicate that biochemical compatibility also plays a role in mammals (e.g., [22-26]), systematic studies of this mechanism have so far been lacking in mouse or human cells. Thus, it is still unknown how widespread such intrinsic compatibility is in mammalian cells, and what drives this compatibility.

In order to address this issue, we systematically tested the compatibility of thousands of combinations of candidate CREs (cCREs) and promoters using MPRAs. We used plasmid-based MPRAs because they are highly scalable [27-29], and because episomal plasmids provide an isolated context that minimises confounding effects of variable chromatin environments and differences in 3D folding. However, so far MPRAs have mostly been used to assess the activity of single elements, either as enhancers or as promoters [27, 30-34], except for one recent study that tested combinations of synthetic elements [29]. To be able to dissect compatibility between enhancers and promoters systematically, we designed cloning strategies that allowed us to test thousands of pairwise cCRE-promoter combinations in different positions and orientations in a reporter plasmid.

As models, we chose three genomic loci of 1-3 $\mathrm{Mb}$ in mouse embryonic stem cells (mESCs). From these loci, which each encompass $\sim 20$ genes, we tested a large fraction of all possible pairwise cCRE-promoter (CCRE-P) combinations. We found that more than half of the active cCREs exhibit significant selectivity for specific subsets of promoters. We dissected some of the underlying sequence determinants. Furthermore, we provide evidence suggesting that 3D folding and intrinsic compatibility are independent mechanisms. Our experimental strategy and datasets provide novel insights into the logic and mechanisms of CCRE-promoter specificity. 
RESULTS

\section{Experimental design}

82 To maximise the probability of testing biologically relevant enhancer-promoter pairs, we combined cCREs and promoters coming from the same region in the genome. We selected three loci of 1-3 Mb in size, each roughly centred around a gene (Nanog, Tfcp2/1 or Klf2) that is key to the control of pluripotency of mESCs. The regulation of these genes is still incompletely understood. In addition, each locus contains about 20 other genes (Figure 1A-C).

For promoters in the regions of interest we included approximately the -350 to $+50 \mathrm{bp}$ segments around all GENCODE-annotated [35] transcription start sites (TSSs). The choice to focus on the range -350 to +50 bp was motivated by our previous study of human promoters, which indicated that most of the relevant information for promoter function is generally contained within this range [30]. This definition of promoters is longer than that of core promoters (which are usually only $\sim 100$ bp long) as was used in most previous enhancer reporter assays [21, 27, $29,32-34,36]$. We considered this to be important, because the extra regulatory information contained in those additional sequences may be relevant for interactions of the promoters with CREs.

Compared to promoters, the annotation of cCREs is much less accurate. However, most cCREs are centred around DNase I hypersensitive sites (DHS) [5, 37, 38]. We therefore selected fragments of $\sim 400$ bp centred around all detected DHS peaks in each locus (Figure 1A-C). This definition of cCREs within the range of typical enhancer definitions [39]. Some authors consider enhancers combinations of multiple DHSs or longer stretches of DNA sequences. However, other studies have shown that the activity of these long enhancers can be reproduced by shorter versions of $\sim 500 \mathrm{bp}[40,41]$. Coordinates of all tested genomic fragments are provided in Supplementary Dataset 1.

We designed two MPRA variants to test many cCRE-P combinations (Figure 1D-E). In the first variant, which we will refer to as Upstream assay, we obtained 82-192 individual cCREs and 18-25 P elements per locus by PCR amplification (Table 1). We pooled all of these fragments and randomly ligated them to form dimer fragments, which we then cloned en masse into a reporter vector, upstream of a randomly barcoded transcription unit that lacked a promoter itself. This resulted into highly complex libraries of cCRE-P, cCRE-cCRE, P-P and P-cCRE pairs, with

110 each individual element in two possible orientations. We then sequenced the libraries to identify 111 the paired fragments, their orientations in the reporter vector, and their linked barcodes. Owing 112 to the simple random ligation step, libraries with tens of thousands of cCRE-P combinations can 113 be obtained with this approach (Table 1 and Supplementary Table 1). Here, we focus on the 114 analysis of cCRE-P pairs, but data from all other configurations are also provided as 115 Supplementary Dataset 2.

116 In a second and complementary approach, we constructed a library in which the cCREs 117 are placed downstream of the reporter gene, i.e., separated $\sim 1 \mathrm{~kb}$ from the promoter (Figure 1E). 118 This was done in two steps: we first cloned a selection of 10 promoters upstream of the barcoded 119 transcription unit, resulting in a set of reporters with different promoters. Next, we inserted a pool 120 of cCREs into this set, downstream of the barcoded reporter unit and in both possible orientations. 
121 We will refer to the assays done with the resulting library as Downstream assay. Due to the two-

122 step cloning protocol, the Downstream assay is less scalable than the Upstream assay, but nevertheless allows for testing of hundreds of cCRE-P combinations (Table 1).

We used all $P$ and cCRE DNA fragments from each of the three loci in separate Upstream assays, whereas we focused on ten promoters and all cCREs from the Klf2 locus in the

126 Downstream assay. Table 1 provides summary statistics of the individual library compositions.

127 Due to the random nature of the combinatorial cloning, we did not recover all possible pairs.

128 Nevertheless, in the three Upstream assays combined we tested a total of 10,678 cCRE-P pairs, 129 or 3,747 pairs if we do not take orientations into account. For the Downstream assay these 130 numbers were 1,364 and 752, respectively. From the Klf2 locus 847 and 676 pairs, respectively, 131 overlapped between the Upstream and Downstream assay. As references, we also inserted each $132 \mathrm{P}$ and $\mathrm{CCRE}$ individually (i.e., unpaired) in the upstream position.

\section{Boost indices estimate promoter-specific activity of cCREs}

135 We then transiently transfected each of these libraries into mESCs. Twenty-four hours 136 after transfection we collected mRNA from the cells, and counted the transcribed barcodes by 137 reverse transcription followed by PCR amplification and high-throughput sequencing. In parallel, 138 barcodes were counted in the plasmid libraries. For each barcode we then normalised the counts 139 in cDNA over the counts detected in the plasmid DNA. Further data processing is described in 140 the Methods. We performed 3 biological replicates per library, which correlated with an average 141 Pearson $r=0.87$ ( 0.83 to 0.90 ) for the Upstream assay and $r=0.98$ (0.98 to 0.99 ) for the 142 Downstream assay. (Figure S1 A-C)

143 We first analysed the transcriptional activities of all singlet (unpaired) $P$ and cCREs in the 144 upstream position. For promoters, these basal activities varied over a $\sim 100$-fold dynamic range 145 (Figure 2A; Figure S2A). Of all cCREs, 40.4\% showed detectable transcriptional activity in the 146 upstream position without any P (Figure 2A; Figure S2A). Such autonomous transcriptional 147 activity is a frequently observed property of enhancers [30,42, 43], and hence these elements 148 are likely to be enhancers. For a few cCREs this activity was as high as some of the strongest promoters, suggesting that they may in fact be un-annotated promoters or very strong enhancers.

We then determined the ability of each CCRE to alter the activity of each linked P. For this, we calculated a boost index for each cCRE-P pair, defined as the $\log _{2}$-fold change in activity

152 of the CCRE-P pair compared to the $P$ element alone. Unexpectedly, 20 negative controls that we included in the Klf2 libraries, consisting of randomly generated DNA sequences of similar size 154 and G/C content as the cCREs, showed a modestly negative boost index (median value -0.45 when inserted upstream) (Figure S1D). This is possibly because lengthening of the reporter constructs alters the topology, supercoiling, transfection efficiency or a combination of these parameters. We therefore corrected all CCRE-P boost indices for this non-specific negative bias

158 (see Methods). After this correction the negative controls had a marginal residual bias (median $\log _{2}$ value -0.19), which we deemed acceptable (Supplementary Figure S1D). 
For each of the three genomic loci, the matrix of corrected boost indices shows a wide diversity of patterns across the cCREs. We observed this both in the Upstream and Downstream assays (Figure 2B-D, Supplementary Figure S2B-D). For example, in the Klf2 locus Upstream assay, cCRE E097 activates most of the tested promoters, while E046 (Figure 2B) and E057 (arrow in Figure 2C) only activate a distinct subset of promoters. Several elements are primarily acting as repressors (e.g, E030 (Figure 2B) and E040, (arrow in Figure 2C)), and some seem neither activating nor repressive (e.g., E070 (Figure 2B) and E085 (arrow in Figure 2C)).

We broadly classified the cCREs according to their overall effects on the linked promoters (Figure S3A). In the Upstream assays, $21 \%$ of cCREs showed positive boost indices that were

171 significantly higher than the rest of cCREs across all tested promoters, indicating that they can act as enhancer elements. About $17 \%$ of the cCREs showed negative boost indices significantly below the rest of CCREs, and hence are putative repressor elements. For the remaining $62 \%$ of cCREs the boost indices across their linked promoters were not significantly higher or lower than the rest; these "ambiguous" elements either have no regulatory effects at all, or they have a mixed repressive/activating/inactive effect that depends on the linked $\mathrm{P}$ (see below).

We were somewhat surprised to identify similar numbers of putative enhancers and repressors, because most annotated cCREs in mammalian genomes are predicted to be enhancers rather than repressive elements [5, 44]. In some cases this repression may be underestimated in our analysis, as the estimates of negative boost indices for lowly active promoters are less reliable due to the higher noise-to-mean ratios at low expression levels (Figure S3B).

For activating elements, the boost indices varied in part according to the basal activities of the cCRE and promoters. Strong boosting occurred primarily at promoters with low basal activities, while highly active promoters were more difficult to boost (FigS3C). This suggests a saturation effect, or it could indicate that promoters with high basal activity are less dependent on distal enhancers. For cCREs, their basal activity is generally a strong positive predictor of their enhancer potency (Fig S3D). However, exceptions to this rule occur, as some cCRE-P pairs show high boost indices even though the basal activity of the cCRE is low (Fig S3D, upper left quadrant).

\section{cCRE effects are predominantly orientation- and position-independent}

193 Next, we asked whether the ability of cCREs to regulate the linked promoters was generally independent of their orientation and position. This was originally posited for enhancers [1], and

195 in some cases also reported for repressive elements [10]. Indeed, in the Upstream assays we 196 found a general positive correlation of the boost indices between the two orientations of the 197 cCREs (Pearson's r=0.68) (Figure S4A). These results are similar to those recently obtained with a minimal core promoter [32]. In the Downstream assay the correlation between orientations

199 was somewhat lower (Pearson's $r=0.47$ ) (Figure S4B). This may be due to the lower dynamic 200 range of the Downstream assay data (Figure S1C). To simplify, for all other analyses we 201 combined the boost indices of + and - orientations of the CCREs by averaging.

We then investigated the degree of position-independence, by comparing the overlapping P-cCRE pairs from the Klf2 locus Downstream and Upstream assays. This showed an overall 
204 Pearson correlation of 0.64 (Figure S4C). We conclude that repressive and activating effects of 205 cCREs are substantially but not completely position-independent, at least for the ten tested 206 promoters from the Klf2 locus.

\section{Extensive selectivity of cCREs for promoters}

208 Visual inspection of the boost index matrices suggested that some cCREs alter the expression of most promoters to similar degrees, while others selectively alter the expression of a subset of

210 promoters. In addition to the examples in Figure 2B from the Klf2 locus, strikingly specific 211 promoter responses to some cCREs are illustrated for the Tfcp2/1 locus in Figure 3A. For 212 example, E060, which forms part of an annotated super-enhancer [45], activates most of the 213 tested promoters, but with boost indices that can vary $>50$-fold between promoters. Two other 214 remarkable examples from the Tfcp2/1 locus are E091 and E096, which each activate only a 215 single, distinct promoters out of the 11-12 promoters that were tested in each instance. Much 216 broader specificity is observed for E064, E073, E074 and E090 from the Nanog locus, which are 217 part of previously identified super-enhancers [46] (Figure S2D).

218 We investigated the degrees of selectivity more systematically. Figure 4A-B depicts the 219 distribution of the boost indices for each cCRE. Clearly, some cCREs have a much broader range 220 of boost indices than others. We used an ANOVA approach with Welch F-test to systematically 221 identify cCREs for which the variance of boost indices was larger than could be explained by 222 experimental noise (see methods). Strikingly, out of 233 cCREs with more than 5 tested cCRE223 P combinations, a total of 139 (59.9\%) (Figure 4B-C) showed significant unexplained variance 224 at an estimated false-discovery rate (FDR) cutoff of 5\%. Thus, at least throughout the three loci 225 that we tested, cCRE-P selectivity is widespread, ranging from strong specificity for one or a few 226 promoters to low specificity as seen in quantitative differences in the regulation of a broad set of 227 promoters.

228 Intersection of the ANOVA-based classification of selective/unselective cCREs with the above broad classification into enhancers and repressors indicates that almost all (94\%) general enhancer elements exhibit significant $P$ selectivity. In contrast, only $34 \%$ of the repressors are detectably biased towards a subset of promoters (Figure 4D). However, we note that this

232 percentage may be underestimated, because at low expression levels the noise levels are higher 233 (Figure S3B). Interestingly, among the "ambiguous" cCREs, 55\% are in fact selective. Such 234 elements mostly activate or repress only very few promoters (e.g., E091 and E096 from the 235 Tfcp2/1 locus; Figure 3) and leave all other promoters unaffected. The remainder of the 236 ambiguous cCREs are probably not functional (e.g., E70 from the Klf2 locus, Figure 2B). In 237 summary, these results indicate that more than half of all tested cCREs exhibits significant 238 preference for specific promoters.

Promoters of housekeeping and developmental genes in Drosophila were reported to 240 have distinct specificities toward cCREs [47]. To investigate whether such a dichotomy could also 241 be observed in our data, we focused on the Klf2 locus, which has roughly equal numbers of 242 housekeeping and non-housekeeping promoters [48] (the Tfcp2/1 and Nanog loci have only three 243 and zero housekeeping genes, respectively). Indeed, hierarchical clustering of the boost index 244 matrix showed a rough separation of the two classes of promoters (Figure S5A). However, this 
245 is largely due to the highly similar cCRE specificities among the housekeeping promoters,

246 whereas the CCRE specificities of the non-housekeeping promoters are much more diverse and 247 generally as distinct from each other as from the housekeeping promoters (Figure S5B). To test 248 whether a housekeeping versus non-housekeeping dichotomy may largely explain our 249 identification of cCREs with significant selectivity (Figure 4B-C), we repeated this analysis after 250 removing all housekeeping promoters. This yielded highly similar results (123 of $221 \mathrm{cCREs}$ are 251 significantly selective at 5\% FDR cutoff, Figure S5C). We conclude that housekeeping promoters 252 may be similarly regulated, but CCRE selectivity goes beyond a simple distinction between 253 housekeeping and non-housekeeping promoters.

\section{Selectivity may be mediated by combinations of multiple TF motifs}

256 Taken together, these results point to a broad spectrum of cCRE specificities for promoters, 257 ranging from largely indiscriminate to highly selective. We searched for sequence motifs that may 258 account for these effects, focusing on binding motifs of transcription factors (TFs) that are 259 expressed in mESCs.

260 We first searched for TF motifs in the cCREs that correlate with boost indices across all promoters. This yielded several dozens of TFs that are candidate activators or repressors (Figure 262 S6A). Several of these, such as Sox2, Nanog, ETV4 and GABPA are known key regulators in mESC cells [49-51]. These TFs may broadly contribute to enhancer activity.

Next, we searched for motifs associated with CCRE-P selectivity. We reasoned that selectivity may be due to certain combinations of TFs bound to CCRE and P. First, we asked whether for any TF the simultaneous presence of its motif at CCRE and P correlated with boost indices (Figure 6SB). This only yielded a weak association of FOXO motifs (at a 5\% FDR cutoff). Possibly this is due to FOXO1, a known regulator in mESCs [52]. We then asked if selectivity may be mediated by multiple TFs rather than single TFs. For this purpose, we took the TF motifs associated with enhancer activity with effect sizes $>0.1(n=66)$ and searched for combinations of motifs that would be associated with higher boost indices if present at both the CCRE and the $P$

272 (Figure 5A-B). This yielded a few dozen stronger associations (at a 1\% FDR cutoff). Some of these associations may be redundant either because of motif similarity or because of motif cooccurrence. For example, the 5 associations between Sox 2 and Klf motifs may represent the KIf4-Sox2 pair (Figure 5B) which are known to cooperate in mESCs [53]. These results indicate that selectivity may be mediated by combinations of multiple TF motifs. Our dataset does not provide sufficient statistical power for an exhaustive search of such combinations.

\section{Chromatin looping is independent of compatibility.}

279 Finally, we considered that certain pairs of cCREs and promoters frequently contact each other 280 in the nucleus, as is indicated by focal or stripe-like enrichment patterns in high-resolution Hi-C 281 maps [54, 55]. While long-range contacts are irrelevant in our MPRAs because the tested 282 elements are directly linked, we asked whether such physical contacts in the native genomic 283 context are related to the selectivity of cCREs for certain promoters according to our MPRAs. We 284 considered two models. In one model, the biochemical interactions that underlie cCRE-P 285 selectivity may promote or stabilise cCRE-P looping interactions. Alternatively, looping 
interactions and cCRE-P selectivity may be independent aspects of cCRE-P interplay that each work by different mechanisms.

To discriminate between these two models, we investigated whether the boost indices of CCRE-P pairs correlate with their contact frequencies in Micro-C, a high-resolution variant of Hi$C$ [55]. Remarkably, we found no correlation between these two quantities (Figure 6A). We also found an extremely weak, although statistically significant, correlation between higher boost indices and longer linear distances of CCRE-P pairs along the genome (Figure 6B).

We conclude that CCRE-P contacts in the nucleus may be independent of their functional compatibility as detected in our reporter assays, raising the interesting possibility that chromatin looping and compatibility are two orthogonal mechanisms of gene regulation.

\section{DISCUSSION}

Only a few other studies have so far attempted to analyse cCRE-P compatibility systematically. An early survey of 27 cCRE-P combinations in human cells did not find evidence for specificity [56], but the assay employed may have been insufficiently quantitative, and the choice of tested elements may have been biased. In contrast, testing of $\sim 200$ cCRE-P pairs in zebrafish pointed to extensive specificity [57]. An MPRA study in Drosophila cells using seven different promoters and genome-wide cCREs suggested that cCRE-P specificity broadly separates between housekeeping and tissue-specific promoters [47]. To our knowledge, our systematic combinatorial testing of cCRE-P combinations in mESCs is the first large-scale study in mammalian cells. The results reveal a broad spectrum of specificities: some cCREs are promiscuous, others are highly specific for certain promoters, and in many instances the specificity is quantitative rather than qualitative. By statistical analysis we found that more than half of the cCREs exhibit a degree of specificity that cannot be explained by experimental noise.

It is likely that cCRE-P compatibility is governed by a complex grammar of TF combinations. Underlying this grammar may be a diversity of molecular mechanisms, including direct and indirect TF-TF interactions [e.g., 53], local concentration of activating factors [33, 58], or functional bridging by cofactors $[21,59]$. Due to the complexity of this grammar, its elucidation may require much larger $c C R E-P$ combinatorial datasets than generated here, as well as systematic mutational analysis $[60,61]$ of individual cCRE-P combinations. Nevertheless, our statistical analysis highlights several candidate combinations of TF motifs that may contribute to the compatibility of some cCRE-P pairs.

Our data indicate that some of the cCREs tested may be repressive elements rather than enhancers even though they were selected from DHSs. This is similar to a recent screen of cCREs in human cells, which identified a large set of candidate repressive elements [62] and to another screen in Drosophila [63]. It will be interesting to further explore the physiological regulatory role of these elements. Particularly to understand their influence on close genes and how repression works in open regions of the genome.

Surprisingly, we found that the boost indices of cCRE-P pairs generally do not correlate 
327 organisation and compatibility are regulated by different mechanisms. We envision that 328 compatibility and 3D organisation may be two independent layers necessary for correct selective 329 gene regulation: 3D organisation such as the formation of chromatin loops and compartments 330 may determine whether CREs and promoters are able to interact, while compatibility may 331 determine whether such an interaction is functional, i.e., gives rise to a change in $\mathrm{P}$ activity.

Our current data were generated with transiently transfected plasmids. Advantages of this approach are that it largely eliminates possible confounding effects of chromatin packaging and

$3343 \mathrm{D}$ folding, and that thousands of CCRE-P combinations could be tested. Even higher throughput 335 combinatorial MPRAs will be useful in order to fully dissect the rules behind compatibility either 336 by testing more CCRE-P combinations or mutagenised cCRE-P pairs. However, further studies 337 are needed to verify and analyse the impact of the observed specificities in the native genomic 338 context. Due to genomic confounding factors, such as chromatin context, 3D organisation, 339 regulatory element redundancy/synergy, and poor scalability, such studies will be challenging 340 and may require the development of new technologies. 
MATERIALS AND METHODS

\section{Selection of cCREs and promoters}

345 For the design of the libraries we selected the cCREs and promoters from three TADs centered 346 around each of the KIf2, Nanog and Tfcp2/1 genes, using TAD coordinates from [54]. cCREs 347 were selected based on DNAse hypersensitivity mapping data from mESCs in both 2i+LIF [38] 348 and serum [5] culturing conditions, which we reprocessed and aligned to the mm10 genome 349 build. DNAse hypersensitivity sites (DHSs) were called using Homer v4.10 with default parameters and peak style "factor". We defined cCREs as 450 bp windows centered on each peak. For promoters we used the Gencode mouse TSS annotation [35]. From each TSS we defined as promoters the $-375+75$ bp region. If the promoter regions overlapped with any CCRE then the promoter was redefined as the $450 \mathrm{bp}$ region surrounding the center of the intersection of both elements. PCR primers were designed for each CCRE and promoter using the batch version of Primer3 (BatchPrimer3 v1.0) [64] allowing for primers to be designed on the 50 bps of each end. This yielded PCR products of $\sim 400 \mathrm{bp}$ for each element.

\section{Upstream assay library generation}

359 For each locus, cCREs and promoters were amplified from mouse genomic DNA (extracted 360 fromE14TG2a mESCs, ATCC CRL-1821) by PCR using My-Taq Red mix (\#BIO-25044; Bioline) 361 in 384 well plates using automated liquid handling (Hamilton Microlab® STAR). PCRs were 362 checked on gel and had a success rate between 60 and $90 \%$ depending on the locus. Equal volumes (10ul) of the resulting PCR products were mixed, and the resulting pool was purified by phenol-chloroform extraction followed by gel purification (BIO-52059; Bioline). The purified DNA fragments were then blunted and phosphorylated using End-It DNA End-Repair Kit (\#ER0720; Epicentre). Part of the repaired pool was set apart for cloning of singlet libraries. The remainder was self-ligated using Fast-link ligase ( $\mathrm{LKO750H}$; Lucigen), after which duplets of $\sim 800 \mathrm{bp}$ were excised from agarose gel and purified (BIO-52059; Bioline). Singlet and duplet pools were A-tailed using using Klenow HC 3' $\rightarrow 5^{\prime}$ exo- (\#M0212L; NEB).

The SURE barcoded vector was prepared as described [30]. Then singlet and duplet pools were separately ligated overnight into the SURE barcoded vector using Takara ligation kit version 2.1 (\#6022; Takara). Ligation products were purified using magnetic bead purification

373 (\#CPCR-0050; CleanNA). Next, $2 \mu$ of the purified ligation products were electroporated into 20 $\mu \mathrm{l}$ of electrocompetent e. cloni $10 \mathrm{G}$ supreme (\#60081-1; Lucigen). Each library was grown

375 overnight in $500 \mathrm{ml}$ of standard Luria Broth (LB) with $50 \mu \mathrm{l} / \mathrm{ml}$ of kanamycin and purified using a maxiprep kit (K210016, Invitrogen).

\section{Downstream assay library generation}

379 The Downstream assay vector was based on a pSMART backbone (Addgene plasmid \# 49157; 380 a gift from James Thomson). It was constructed using standard molecular biology techniques 381 and contains a green fluorescent protein (GFP) open reading frame followed by a barcode, and 382 a psiCheck polyadenylation signal (PAS) introduced during barcodin, followed by the cloning 383 site for inserts and a triple polyadenylation site (SV40+bGH+psiCheckPAS). 
The 10 highest expressing promoters of the KIf2 Upstream library were selected to be cloned into the Downstream assay vector at the promoter position. These Promoters were amplified by PCR and individually inserted by Gibson assembly (\#E2611S; NEB) into the Downstream assay vector. Then each of the 10 constructs were transformed into standard DH5a competent bacteria (\#C2987; NEB) grown overnight in in $500 \mathrm{ml}$ of standard Luria Broth(LB) with $50 \mu \mathrm{l} / \mathrm{ml}$ of kanamycin and purified.

Each of these promoter-containing vectors was then barcoded similarly as the SURE vector [30]. For this, we digested $10 \mu \mathrm{g}$ of each vector with Avrll (\#ER1561; Thermo Fischer) and Xcml (\#R0533; NEB) and performed a gel-purification. Barcodes were generated by performing $10 \mathrm{PCR}$ reactions of $100 \mu \mathrm{l}$ each containing $5 \mu \mathrm{l}$ of $10 \mu \mathrm{M}$ primer 275JvA, $5 \mu \mathrm{l}$ of 10 $\mu \mathrm{M}$ primer 465JvA and $1 \mu \mathrm{l}$ of $0.1 \mu \mathrm{M}$ template 274JvA (see Supplementary Table 2 for

395 oligonucleotide sequences). A total of 14 PCR cycles were performed using MyTaq Red Mix (\#BIO-25043; Bioline), yielding 30 $\mu \mathrm{g}$ barcodes. Barcodes were purified by phenol-chloroform extraction and isopropanol precipitation after which they were digested overnight with 80 units of Nhel (\#R0131S; NEB) and purified using magnetic bead purification (\#CPCR-0050; CleanNA). Each vector variant and the barcodes were then ligated in one $100 \mu \mathrm{l}$ reaction containing $3 \mu \mathrm{g}$ digested vector and $2.7 \mu \mathrm{g}$ digested barcodes, 20 units Nhel (\#R0131S; NEB), 20 units Avrll, $10 \mu \mathrm{l}$ of $10 \times$ CutSmart buffer, $10 \mu \mathrm{l}$ of $10 \mathrm{mM}$ ATP, 10 units T4 DNA ligase (\#10799009001 Roche). A cycle-ligation of six cycles was performed $\left(10 \mathrm{~min}\right.$ at $22{ }^{\circ} \mathrm{C}$ and 10 min at $37^{\circ} \mathrm{C}$ ), followed by 20 min heat-inactivation at $80^{\circ} \mathrm{C}$. The ligation reaction was purified by magnetic beads and digested with 40 units of Xcml (\#R0533S; NEB) for $3 \mathrm{~h}$, and sizeselected by gel-purification, yielding $\sim 1 \mu \mathrm{g}$ barcoded vector for each variant.

\section{Inverse PCR and sequencing to link inserted elements to barcodes}

408 We identified barcode-insert combinations in the plasmid libraries by inverse-PCR followed by sequencing as described [30]. In brief, the combination of barcode and element(s) was excised from the plasmid by digestion with I-ceul; this fragment was circularised; remaining linear fragments were destroyed; and circular fragments were linearised again with I-scel. These linear fragments were amplified by PCR with sequencing adaptors. The final product was sequenced on an Illumina MiSeq platform using $150 \mathrm{bp}$ paired-end reads. This process was done separately for each of the libraries. In the singlet libraries the barcodes should be associated to only one insert and in the combinatorial libraries the barcodes should be associated with duplets.

\section{Linking barcodes to element singlets or duplets}

419 For each library the iPCR data was locally aligned using bowtie (version 2.3.4) [65] with very sensitive parameters (--very-sensitive-local) on a custom bowtie genome. This custom genome was generated using bowtie. It consists of virtual chromosomes corresponding to each cCRE or a $P$ from each locus. Bam alignment files were processed using a custom python script that identifies from read 1 the barcode and CCRE or $\mathrm{P}$ element, and from read 2 the cCRE or $\mathrm{P}$ element. In case of singlet libraries both reads should identify the same element, whereas in 
425 combinatorial libraries read 1 is derived from the barcode-proximal element and read 2 from the

426 barcode distal element. In the combinatorial libraries we can not distinguish between a

427 combination of one element with itself in the same orientation or a single element, therefore

428 these were removed from combinatorial libraries. In the Downstream Assay both reads identify

429 the only element cloned in the downstream position. If no element was found, the barcode was

430 assigned as empty vector. The resulting barcode-to-element(s) lists were clustered using

431 Starcode (version 1.1) [66] to remove errors from barcode sequencing. Finally, barcodes

432 present in multiple libraries or matched with multiple element combinations were removed from

433 the data.

\section{Cell culture and transfection}

436 All experiments were conducted in E14TG2a mouse embryonic stem cells (mESC) (ATCC

437 CRL-1821) cultured in 2i+LIFulturing media. 2i+LIF was made according to the 4DN nucleome

438 protocol for culturing mESCs (https://data.4dnucleome.org/protocols/cb03c0c6-4ba6-4bbe-

439 9210-c430ee4fdb2cl). The reagents used were Neurobasal medium (\#21103-049, Gibco),

440 DMEM-F12 medium (\#11320-033, Gibco), BSA (\#15260-037; Gibco), N27 (\#17504-044;

441 Gibco), B2 (\#17502-048; Gibco), LIF(\#ESG1107; Sigma-Aldrich), CHIR-99021 (\#HY-10182;

442 MedChemExpress) and PD0325901 (\#HY-10254; MedChemExpress), monothioglycerol

443 (\#M6145-25ML; Sigma) and glutamine (\#25030-081, Gibco). Monthly tests (\#LT07-318; Lonza)

444 confirmed that the cells were not contaminated by mycoplasma. Cells were transiently

445 transfected using Amaxa nucleofector II, program A-30, and Mouse Embryonic Stem Cell

446 Nucleofector ${ }^{T M}$ Kit (\#VPH-1001, Lonza). KIf2 and Nanog loci Upstream assay libraries were

447 mixed and transfected together, Tfcp2/1 Upstream Assay libraries were transfected in separate

448 experiments. All the Downstream assay sub-libraries were transfected as a mix. Three

449 independent biological replicates were done for each library mix. For each biological replicate

45016 million cells were transfected ( 4 million cells with $4 \mu \mathrm{g}$ plasmid per cuvette)

\section{RNA extraction and cDNA sequencing}

453 RNA was extracted and processed for sequencing as described [30] with a few modifications.

454 Cells were harvested $24 \mathrm{~h}$ after transfection, resuspended in Trisure (\#BIO-38032; Bioline) and

455 frozen at $-80^{\circ} \mathrm{C}$ until further processing. From the Trisure suspension, the aqueous phase

456 containing the RNA was extracted and loaded into RNA extraction columns (\#K0732, Thermo

457 Scientific). Total RNA was divided into $10 \mu \mathrm{l}$ reactions containing $5 \mu \mathrm{g}$ of RNA and was treated

458 for 30 mins with 10 units of DNAse I (\#04716728001; Roche). Then DNAse I was inactivated by 459 addition of $1 \mu \mathrm{l}$ of $25 \mathrm{mM}$ EDTA and incubation at $70^{\circ} \mathrm{C}$ for $10 \mathrm{~min}$.

$460 \quad$ For the Upstream Assay the cDNA was produced and amplified by PCR as described

461 [30]. Per biological replicate 8 to 10 reactions were carried out in parallel in order to cover

462 enough barcode complexity of the library. For the Downstream Assay the RNA was extracted

463 and processed the same way until cDNA production Here, cDNA was produced using a specific

464 primer (304JvA sequence in Supplementary Table 2 for oligonucleotide sequences). Primer

465 304JvA introduces an adaptor sequence 5' to the primer sequence which is targeted in the first 
466 PCR (see below) to ensure strand specific amplification of barcodes. Then cDNA was amplified 467 in 2 steps (nested PCRs) in order to make the reaction strand-specific. The first $\mathrm{PCR}$ reaction 468 was run for 10 cycles $\left(1 \mathrm{~min} 96^{\circ} \mathrm{C}, 10\right.$ times $\left(15 \mathrm{~s} 96^{\circ} \mathrm{C}, 15 \mathrm{~s} 60^{\circ} \mathrm{C}, 15 \mathrm{~s} 72{ }^{\circ} \mathrm{C}\right)$ ) using (index 469 variants of) primers $285 \mathrm{JvA}$ (containing the S2, index and $\mathrm{p} 7$ adaptor) and 305JvA (targeting 470 the adapter introduced by 304JvA). Each $20 \mu \mathrm{LT}$ reaction was amplified in a 100- $\mu \mathrm{I} P C R$ 471 reaction with MyTaq Red mix. The second PCR reaction was performed using 10ul of the 472 product of the previous reaction in $100 \mu \mathrm{l}$ reactions $\left(1 \mathrm{~min} 96^{\circ} \mathrm{C}, 8 \times\left(15 \mathrm{~s} 96^{\circ} \mathrm{C}, 15 \mathrm{~s} 60^{\circ} \mathrm{C}, 15\right.\right.$ $473 \mathrm{~s} 72^{\circ} \mathrm{C}$ )) using the same index variant primer and primer 437JvA (containing the S1, and p5 474 adaptor). For both Upstream and Downstream assays, the resulting PCR products were 475 sequenced on an Illumina 2500 HiSeq platform with $65 \mathrm{bp}$ single end reads.

\section{Plasmid DNA (pDNA) barcode sequencing}

478 For normalisation purposes, barcodes in the plasmid pools were counted as follows. For both assays the process was the same. For each library $1 \mu \mathrm{g}$ of plasmid was digested with I-scel in order to linearise the plasmid. Then, barcodes were amplified by PCR from $50 \mathrm{ng}$ of material using the same primers and reaction conditions as in the amplification of cDNA in the Upstream assay, but only 9 cycles of amplification were used $\left(1 \min 96{ }^{\circ} \mathrm{C}, 9\right.$ times $\left(15 \mathrm{~s} 96{ }^{\circ} \mathrm{C}, 15 \mathrm{~s} 60\right.$ $\left.{ }^{\circ} \mathrm{C}, 15 \mathrm{~s} 72^{\circ} \mathrm{C}\right)$ ). For each library, two technical replicates were carried out by using different index primers for each replicate. Samples were sequenced on an Illumina $2500 \mathrm{HiSeq}$ platform with $65 \mathrm{bp}$ single end reads.

\section{Pre-Processing of $c D N A$ and $p D N A$ reads}

488 For each replicate of each library pool transfection barcodes were extracted from the single end reads by using a custom python script that identifies the constant region after the barcode. Near-identical barcodes were pooled using Starcode (version 1.1) [66] to remove errors from barcode sequencing, and barcode counts were summarised. The process was the same for cDNA and pDNA counts and for Upstream and Downstream data.

\section{Post processing of $c D N A$ and pDNA counts}

495 For each transfection, barcodes identified in the cDNA were matched to the barcodes in the iPCR data, and all barcodes were counted in cDNA and pDNA replicates. Barcode counts were normalised to the total number of barcode reads from each sample. Activity per barcode was then calculated as a cDNA:pDNA ratio of normalised counts. Next, activities from multiple barcodes belonging to the same element singlet or combination were averaged, requiring a minimum of 5 barcodes per singlet or combination and at least 8 pDNA counts per barcode.

501 The mean activity of each singlet or combination across replicates was calculated as the geometric mean of the three replicates.

\section{Calculation of boost indices}

505 We initially calculated raw boost indices simply as a $\log _{2}$ ratio of the activity of each cCRE-P 506 pair over the activity of the corresponding $P$ alone. However, 20 negative controls that we 507 included in the KIf2 libraries, consisting of randomly generated DNA sequences of similar size 
508 and G/C content as the cCREs (Supplementary dataset 1), generally showed a negative

509 boost index by this measure (median value -0.45 when inserted upstream) (Figure S1D). We

510 therefore calculated corrected boost indices as the $\log _{2}$ ratio of cCRE-P activity over the median

511 cCRE-P activity per promoter (Figure S1D). Importantly, in the KIf2 library data this largely

512 removed the negative bias that we observed with the negative controls; we thus assume that

513 this correction is adequate and therefore also applied it to the boost indices obtained with the

514 other libraries. For the analyses in Figures 2-6 and Supplementary figures 2-6 except 4A-B

515 the boost indices of cCREs were averaged over both orientations of the cCREs.

\section{Analysis of selectivity}

518 We performed a Welch's ANOVA (or Welch F-test) to assess the selectivity of each cCRE with 519 more than 5 CCRE-P combinations. For this purpose, each replicate of each orientation of the 520 cCRE-P was used as a datapoint and each cCRE-P combination was used as a group. P-

521 values were corrected for multiple hypothesis testing using the Benjamini-Hochberg method 522 and an FDR cutoff of $5 \%$ was chosen. The Welch F-test was chosen over the classic ANOVA 523 due to heteroscedasticity of the data.

526 We used a custom TF motif database provided by the lab of Gioacchino Natoli containing 2,448

527 TF motifs which was built on top of a previously published version [67] (Dataset composition 528 and sources available at \#\#GitHub-url). TF motifs were filtered for expression of TFs in mESCs 529 cultured in 2i+LIF according to published RNA-seq (higher expression than 1RPM) [38]. We 530 scored presence or absence of a TF motif in each CCRE using FIMO (MEME suite, version 531 5.0.2). We then searched for motifs associated with (1) general enhancer activity, (2) self532 compatibility and (3) duplets of self-compatible motifs. In (1), for each TF motif we compared 533 the general CCRE-P population to combinations where the TF motif was present at the cCRE.

534 In (2), for each TF motif we compared the cCRE-P combinations where the TF motif was 535 present at the CCRE to the combinations where it was present at both the CCRE and the 536 promoter. In (3), we took all the significant TF motifs at a 1\% FDR and an effect size higher 537 than $0.1(n=66)$. Then we tested all pairwise non-repeated TF motif duplets. Per TF motif duplet 538 we compared the CCRE- promoters where both TF motif were present at the cCRE to the 539 combinations where both were present at both the CCRE and the promoter. In all comparisons 540 a Wilcoxon test was applied to the boost indices of each group and the effect size was 541 calculated a difference of median boost indices. In each analysis p-values were corrected for 542 multiple hypothesis testing using the Benjamini-Hochberg method. We required a minimum of 54350 combinations per group.

\section{Micro-C data correlation}

546 Micro-C data was obtained from [55]. Contact scores between cCRE-P pairs were averaged 547 across bins overlapping a +-500 bp window from the location of each element using $400 \mathrm{bp}$ 548 bins. 
bioRxiv preprint doi: https://doi.org/10.1101/2021.10.21.465269; this version posted October $22,2021$. The copyright holder for this preprint (which was not certified by peer review) is the author/funder, who has granted bioRxiv a license to display the preprint in perpetuity. It is made available under aCC-BY-NC-ND 4.0 International license.

\section{Data analysis and data availability.}

551 All data analysis was performed in R [68]. Code of data processing pipelines and analysis

552 scripts are available at \#Github-url. Raw and processed data are available at GEO (accession

$553 \mathrm{nr}$ GSE186265). Processed datasets and pipeline output files are available at OSF (\#\#OSF-ur).

554

555

556 


\section{Author contributions}

559 M.M.A, F.C. and B.v.S. designed the study. M.M.A and F.C. developed computational methods

560 and performed analyses. M.M.A. and J.v.A. developed experimental methods. M.M.A.

561 performed experiments. B.v.S. and M.M.A. wrote the manuscript, with input from F.C. and

562 J.v.A. B.v.S. supervised the study.

563

\section{Acknowledgements}

565 We thank Tao Chen for initial input on the study design, the NKI Genomics, Robotics and

566 Research High Performing Computing facilities for technical support, Barak Cohen and his lab

567 for insightful discussions, and Gioacchino Natoli and his lab for providing the TF motif

568 database. Supported by ERC Advanced Grant 694466 (B.v.S.) and Swiss National Science

569 Foundation postdoctoral fellowship P2EZP3_165206 (F.C). Oncode is partly funded by the

570 Dutch Cancer Society KWF.

\section{Competing Interests}

573 J.v.A. is founder of Gen-X B.V. and Annogen B.V. F.C. is a co-founder of enGene Statistics $574 \mathrm{GmbH}$. 


\section{FIGURE LEGENDS}

577 Figure 1. Regulatory element selection and library construction. A-C) Representations of

578 Nanog, Tfcp2/1, and KIf2 loci, respectively. In C) the zoom-in displays a DNAse I sensitivity

579 track [38] where peaks overlap with cCREs. D) Cloning strategy for the Upstream assay.

580 cCREs and promoters were amplified by PCR from genomic DNA and pooled. Fragments in

581 this pool were then randomly ligated to generate duplets. Singlets and duplets were cloned into

582 the same barcoded vector to generate two libraries per locus, a singlet library and a

583 combinatorial library. E) Cloning strategy for the Downstream assay. The singlet pool from the

584 Klf2 locus was cloned into ten vectors, each of them carrying a different promoter. The resulting

585 ten sub-libraries were combined into one Downstream assay library.

587 Figure 2. Singlet and combinatorial activities of cCREs and promoters from the KIf2 locus. A)

588 Transcription activities of singlet cCREs and promoters. Each dot represents the mean activity

589 of one singlet. Horizontal lines represent the average background activity of empty vectors

590 (black line) plus or minus two standard deviations (grey lines). Elements with activities more

591 than two standard deviations above the average background signal are defined as active. B)

592 Examples of Upstream assay cCRE-P combinations for cCREs E097, E046, E030 and E070 of

593 the KIf2 locus. Barplots represent the mean boost index of each combination, vertical lines

594 represent the standard deviations. Crosses mark missing data. C-D) Boost index matrices of

595 cCRE-P combinations from the KIf2 locus according to Upstream (C) and Downstream (D)

596 assays. White tiles indicate missing data. Barplots on the right and top of each panel show

597 basal activities of each tested P or CCRE, respectively, with the black line indicating the

598 background activity of the empty vector. All data are averages over 3 independent biological

599 replicates.

Figure 3. Examples of selective cCREs from the Tfcp2/1 locus. Boost indices obtained in the

602 Upstream assay are shown for CCRE-P combinations of CCREs E060, E091 and E096 of the

603 Tfcp2/1 locus. Barplots indicate the mean boost index of each combination, vertical lines

604 indicate standard deviations. All data are averages over 3 independent biological replicates.

Figure 4. Promoter selectivity of cCREs. A) Plot showing the broad diversity of boost indices of many cCREs. Data are from Upstream assays of Klf2, Nanog and Tfcp2/1 loci combined.

608 Vertical axis indicates boost indices of all tested cCRE-P pairs, which are horizontally ordered

609 by the mean boost index of each CCRE. B) Boost index distributions for each cCRE from the

610 Klf2 locus (Upstream assay). Each dot represents one cCRE-P combination; black bar

611 represents the mean. Turquoise colouring marks cCREs that have a larger variance of their

612 boost indices than may be expected based on experimental noise, according to the Welch F-

613 test after multiple hypothesis correction (5\% FDR cutoff). C) Summary of Welch F-test

614 selectivity analysis results for all cCREs from the three loci with more than 5 cCRE-P

615 combinations. Each dot represents one cCRE; the size of the dots indicates the number of

616 cCRE-P pairs. Significantly selective cCREs (5\% FDR cutoff) are highlighted in turquoise. D) 
617 Proportion of significantly selective (turquoise) CCRE in the three categories as shown in

618 Figure S3A. All data are averages over 3 independent biological replicates.

Figure 5. Association of TF motif Duos with higher boost indices. A) Results of TF survey for self-compatible TF motif Duos. TF motif duos associated with higher or lower boost indices at a 1\% FDR cutoff are highlighted. B) Association of Sox2+KIf4 motifs at both cCRE and P with higher boost indices. cCRE-P combinations are split into 3 groups according to presence or absence of Sox2+KIf4 motifs both at the cCRE and the promoter, or only the cCRE. Numbers at the top of horizontal brackets are the $p$-values obtained from comparing the different groups boost index distributions using a Wilcoxon rank-sum test. Boxplots represent median and interquartile ranges. Barplots at the top represent the number of combinations in each group.

Figure 6. Absent or very weak correlation between boost indices and (A) contact frequencies according to micro-C [55] or (B) linear genomic distance, for all cCRE-P pairs from the three loci combined. All boost index data are averages over 3 independent biological replicates.

\section{SUPPLEMENTARY FIGURE LEGENDS}

Figure S1. Reproducibility of data and boost index calculation. (A-C) Correlograms of the three biological replicates of each library pool. Lower left panels show pairwise scatterplots of the activities of all CCRE-P pairs per replicate. Middle panels show the density of data distribution in each replicate and upper right panels show the Pearson correlation coefficients. A) KIf2 and Nanog Upstream libraries. B) Tfcp2/1 Upstream library. C) KIf2 Downstream libraries. D) Upstream assay boost index distributions for CCRE-P and negative controls - promoter (NC-P) combinations. Left panel: raw boost indices; right panel: boost indices after correction for negative bias (see Methods).

Figure S2. Element activities and boost indices obtained with Nanog and Tfcp2/1 Upstream libraries. A) Transcriptional activities of CCREs and promoters. Each dot represents the mean activity of one singlet. Horizontal lines represent the average background activity of empty vectors (black line) plus or minus two standard deviations (grey lines). Elements with activities more than two standard deviations above the average background signal are defined as active. B-C) Boost index matrices for cCRE-P pairs from Nanog and Tfcp2/1 loci (both Upstream assays). White tiles indicate missing data. Barplots on the right and top of each panel show basal activities of each tested $P$ or CCRE, respectively, with the black line indicating the background activity of the empty vector. D) Examples of cCRE-P combinations for cCREs E064, E073, E074 and E090 of the Nanog locus. Barplots represent the mean boost index of each combination, vertical lines represent the standard deviation of each boost index. All data are averages over 3 independent biological replicates. 
659 Figure S3. cCRE functional classification and activity influence on Boost indices. A) Volcano 660 plot of cCREs associated with activation or repression across promoters. A Wilcoxon test is 661 performed per cCRE comparing the boost indices of all the cCRE-P combinations of that CCRE 662 against the rest of CCRE-P combinations. A minimum of 6 combinations is required per cCRE. 663 P-values are corrected for multiple hypothesis testing using the Benjamini-Hochberg method 664 (FDR). B) Relationship between noise-to-mean ratio (Standard Deviation/mean Activity) and 665 mean activity of cCRE-Ps. Horizontal lines represent noise-to-mean ratios of 1 and of 4 in log2 666 scale. C) Relationship between boost indices and basal (singlet) P activity. Each column of dots 667 shows the data of CCRE-P pairs for one P. Data are from Upstream assays of all three loci 668 combined. D) Relationship between boost indices and basal (singlet) cCRE activity. All data are averages over 3 independent biological replicates.

Figure S4. Orientation and position independence of cCREs. (A-B) Correlation between boost indices of both cCRE orientations of the same cCRE-P combination, in the (A) Upstream assay and (B) Downstream assay. Data are from the Klf2 locus libraries. Note that "+" and "-" orientations are arbitrary labels, because cCREs do not have an intrinsic orientation. (C) Correlation between boost indices of CCRE-P combinations shared between the Upstream and Downstream assays of the KIf2 locus. In all panels $\mathrm{R}$ is the Pearson correlation coefficient. All data are averages over 3 independent biological replicates. In C Boost indices are averaged over cCRE orientations.

Figure S5. Housekeeping promoters show a distinct pattern of cCRE compatibility. A) Hierarchical clustering of the Upstream assay boosting matrix of the KIf2 locus. In order to facilitate hierarchical clustering the matrix has been restricted to almost complete cases (cCREs >15 combinations) B) Density plot of pairwise Pearson correlation coefficients of the boost indices of KIf2 locus promoters classified as either housekeeping or non-housekeeping [48]. Blue: correlations between all pairs of housekeeping promoters; red: all correlations between pairs of non-housekeeping promoters; grey: all correlations between one housekeeping and one non-housekeeping promoter. Vertical lines represent the median of each group. Unlike in (A), all promoters in the Upstream assay were included in this analysis. C) Results of selectivity analysis as performed in Figure 4C, but excluding housekeeping promoters. All data are averages over 3 independent biological replicates.

Figure S6. Identification of single TF motifs that correlate with boost indices. (A) TF motifs in cCREs associated (at 1\% FDR cutoff) with activation (turquoise) or repression (red). (B) Motifs of putative self-compatible TFs, i.e. motifs that predict increased or reduced boosting indices when present both at the CCRE and $\mathrm{P}$, compared to being present only at the CCRE. TF motifs associated with higher or lower boost indices at a 1\% FDR cutoff are highlighted. We note that 
TF motifs with multiple hits from the same family, such as for ELK, FOXO and ELF factors, may in fact be due to the activity of one TF motif of that family [69].

\section{TABLES}

Table 1. Numbers of tested Promoters (Ps), cCREs and cCRE-P pairs in each combinatorial MPRA library.

\begin{tabular}{|l|l|l|l|l|}
\hline Library & Ps present & $\begin{array}{l}\text { cCREs } \\
\text { present }\end{array}$ & $\begin{array}{l}\text { cCRE-P } \\
\text { pairs tested }\end{array}$ & cCRE-P pairs (orientation-independent) \\
\hline Klf2 Upstream & 23 & 82 & 3758 & 1400 \\
\hline Nanog Upstream & 18 & 88 & 1321 & 595 \\
\hline Tfcp2I1 Upstream & 25 & 198 & 5599 & 2490 \\
\hline Klf2 Downstream & 10 & 84 & 1364 & 752 \\
\hline
\end{tabular}

\section{SUPPLEMENTARY TABLES}

Supplementary table 1. Other combinations of cCRE and P elements in each MPRA library.

713

714

715

716

\begin{tabular}{|l|l|l|l|l|l|l|}
\hline Library & $\begin{array}{l}\text { cCRE- } \\
\text { cCRE }\end{array}$ & $\begin{array}{l}\text { CCRE-cCRE } \\
\text { (orientation- } \\
\text { independent) }\end{array}$ & P-P & $\begin{array}{l}\text { P-P } \\
\text { (orientation- } \\
\text { independent) }\end{array}$ & P-CCRE & $\begin{array}{l}\text { P-cCRE } \\
\text { (orienta- } \\
\text { tion-inde- } \\
\text { pendent) }\end{array}$ \\
\hline KIf2 Upstream & 10626 & 4284 & 1335 & 441 & 4067 & 1439 \\
\hline Nanog Upstream & 10536 & 4769 & 155 & 82 & 1511 & 713 \\
\hline Tfcp211 Upstream & 44515 & 21149 & 626 & 274 & 5239 & 2386 \\
\hline KIf2 Downstream & 0 & 0 & 420 & 225 & 0 & 0 \\
\hline
\end{tabular}

Supplementary table 2. Oligonucleotide and plasmid sequences (supplementary file)

\section{SUPPLEMENTARY DATASETS}

\section{Data Set 1 Coordinates and sequences of cCREs and Promoters}

Data Set 2 Activities of all cCRE-cCRE, cCRE-P, P-cCRE and P-P combinations Upstream assay

Data Set 3 Boost indices of cCRE-P combinations Upstream assay

Data Set 4 Boost indices of cCRE-P combinations Downstream assay 


\section{REFERENCES}

1. Banerji, J., S. Rusconi, and W. Schaffner, Expression of a beta-globin gene is enhanced by remote SV40 DNA sequences. Cell, 1981. 27(2 Pt 1): p. 299-308.

2. Tuan, D., et al., The "beta-like-globin" gene domain in human erythroid cells. Proc Natl Acad Sci U S A, 1985. 82(19): p. 6384-8.

3. Fiering, S., et al., Targeted deletion of $5^{\prime} H S 2$ of the murine beta-globin LCR reveals that it is not essential for proper regulation of the beta-globin locus. Genes Dev, 1995. 9(18): p. 2203-13.

4. Lettice, L.A., et al., A long-range Shh enhancer regulates expression in the developing limb and fin and is associated with preaxial polydactyly. Hum Mol Genet, 2003. 12(14): p. $1725-35$.

5. Consortium, E.P., An integrated encyclopedia of DNA elements in the human genome. Nature, 2012. 489(7414): p. 57-74.

6. van Arensbergen, J., B. van Steensel, and H.J. Bussemaker, In search of the determinants of enhancer-promoter interaction specificity. Trends Cell Biol, 2014. 24(11): p. 695-702.

7. Zabidi, M.A. and A. Stark, Regulatory Enhancer-Core-Promoter Communication via Transcription Factors and Cofactors. Trends Genet, 2016. 32(12): p. 801-814.

8. Farley, E.K., K.M. Olson, and M.S. Levine, Regulatory Principles Governing Tissue Specificity of Developmental Enhancers. Cold Spring Harb Symp Quant Biol, 2015. 80: p. 27-32.

9. Robson, M.I., A.R. Ringel, and S. Mundlos, Regulatory Landscaping: How EnhancerPromoter Communication Is Sculpted in 3D. Mol Cell, 2019. 74(6): p. 1110-1122.

10. Segert, J.A., S.S. Gisselbrecht, and M.L. Bulyk, Transcriptional Silencers: Driving Gene Expression with the Brakes On. Trends Genet, 2021. 37(6): p. 514-527.

11. Shlyueva, D., G. Stampfel, and A. Stark, Transcriptional enhancers: from properties to genome-wide predictions. Nat Rev Genet, 2014. 15(4): p. 272-86.

12. Schoenfelder, S. and P. Fraser, Long-range enhancer-promoter contacts in gene expression control. Nat Rev Genet, 2019. 20(8): p. 437-455.

13. Furlong, E.E.M. and M. Levine, Developmental enhancers and chromosome topology. Science, 2018. 361(6409): p. 1341-1345.

14. Lupianez, D.G., et al., Disruptions of topological chromatin domains cause pathogenic rewiring of gene-enhancer interactions. Cell, 2015. 161(5): p. 1012-1025.

15. $\mathrm{Li}, \mathrm{X}$. and M. Noll, Compatibility between enhancers and promoters determines the transcriptional specificity of gooseberry and gooseberry neuro in the Drosophila embryo. EMBO J, 1994. 13(2): p. 400-6.

16. Merli, C., et al., Promoter specificity mediates the independent regulation of neighboring genes. Genes Dev, 1996. 10(10): p. 1260-70.

17. Butler, J.E. and J.T. Kadonaga, Enhancer-promoter specificity mediated by DPE or TATA core promoter motifs. Genes Dev, 2001. 15(19): p. 2515-9.

18. Juven-Gershon, T., J.Y. Hsu, and J.T. Kadonaga, Caudal, a key developmental regulator, is a DPE-specific transcriptional factor. Genes Dev, 2008. 22(20): p. 2823-30.

19. Kwon, D., et al., Enhancer-promoter communication at the Drosophila engrailed locus. Development, 2009. 136(18): p. 3067-75.

20. Arnold, C.D., et al., Genome-wide assessment of sequence-intrinsic enhancer responsiveness at single-base-pair resolution. Nat Biotechnol, 2017. 35(2): p. 136-144. 
21. Haberle, V., et al., Transcriptional cofactors display specificity for distinct types of core promoters. Nature, 2019. 570(7759): p. 122-126.

22. Bertolino, E. and H. Singh, POU/TBP cooperativity: a mechanism for enhancer action from a distance. Mol Cell, 2002. 10(2): p. 397-407.

23. Vakoc, C.R., et al., Proximity among distant regulatory elements at the beta-globin locus requires GATA-1 and FOG-1. Mol Cell, 2005. 17(3): p. 453-62.

24. Jing, H., et al., Exchange of GATA factors mediates transitions in looped chromatin organization at a developmentally regulated gene locus. Mol Cell, 2008. 29(2): p. 23242.

25. Deng, W., et al., Controlling long-range genomic interactions at a native locus by targeted tethering of a looping factor. Cell, 2012. 149(6): p. 1233-44.

26. Chang, T.H., et al., An enhancer directs differential expression of the linked Mrf4 and Myf5 myogenic regulatory genes in the mouse. Dev Biol, 2004. 269(2): p. 595-608.

27. Inoue, $\mathrm{F}$. and $\mathrm{N}$. Ahituv, Decoding enhancers using massively parallel reporter assays. Genomics, 2015. 106(3): p. 159-164.

28. van Arensbergen, J., et al., High-throughput identification of human SNPs affecting regulatory element activity. Nat Genet, 2019. 51(7): p. 1160-1169.

29. Sahu, B., et al., Sequence determinants of human gene regulatory elements. bioRxiv, 2021: p. 2021.03.18.435942.

30. van Arensbergen, J., et al., Genome-wide mapping of autonomous promoter activity in human cells. Nat Biotechnol, 2017. 35(2): p. 145-153.

31. Arnold, C.D., et al., Genome-wide quantitative enhancer activity maps identified by STARR-seq. Science, 2013. 339(6123): p. 1074-7.

32. Klein, J.C., et al., A systematic evaluation of the design and context dependencies of massively parallel reporter assays. Nat Methods, 2020. 17(11): p. 1083-1091.

33. Davis, J.E., et al., Dissection of C-AMP Response Element Architecture by Using Genomic and Episomal Massively Parallel Reporter Assays. Cell Syst, 2020. 11(1): p. 75-85 e7.

34. King, D.M., et al., Synthetic and genomic regulatory elements reveal aspects of cisregulatory grammar in mouse embryonic stem cells. Elife, 2020. 9.

35. Frankish, A., et al., GENCODE reference annotation for the human and mouse genomes. Nucleic Acids Res, 2019. 47(D1): p. D766-D773.

36. Ohler, U., et al., Computational analysis of core promoters in the Drosophila genome. Genome Biol, 2002. 3(12): p. RESEARCH0087.

37. Groudine, M., et al., Human fetal to adult hemoglobin switching: changes in chromatin structure of the beta-globin gene locus. Proc Natl Acad Sci U S A, 1983. 80(24): $p$. 7551-5.

38. Joshi, O., et al., Dynamic Reorganization of Extremely Long-Range Promoter-Promoter Interactions between Two States of Pluripotency. Cell Stem Cell, 2015. 17(6): p. 748757.

39. Long, H.K., S.L. Prescott, and J. Wysocka, Ever-Changing Landscapes: Transcriptional Enhancers in Development and Evolution. Cell, 2016. 167(5): p. 1170-1187.

40. Barakat, T.S., et al., Functional Dissection of the Enhancer Repertoire in Human Embryonic Stem Cells. Cell Stem Cell, 2018. 23(2): p. 276-288 e8.

41. Agrawal, P., et al., Genome editing demonstrates that the $-5 \mathrm{~kb}$ Nanog enhancer regulates Nanog expression by modulating RNAPII initiation and/or recruitment. J Biol Chem, 2021. 296: p. 100189.

42. Djebali, S., et al., Landscape of transcription in human cells. Nature, 2012. 489(7414): p. 101-8. 
bioRxiv preprint doi: https://doi.org/10.1101/2021.10.21.465269; this version posted October $22,2021$. The copyright holder for this preprint (which was not certified by peer review) is the author/funder, who has granted bioRxiv a license to display the preprint in perpetuity. It is made available under aCC-BY-NC-ND 4.0 International license.

822 43. Andersson, R., et al., An atlas of active enhancers across human cell types and tissues. Nature, 2014. 507(7493): p. 455-461.

44. Consortium, E.P., et al., Expanded encyclopaedias of DNA elements in the human and mouse genomes. Nature, 2020. 583(7818): p. 699-710.

45. Khan, A. and X. Zhang, dbSUPER: a database of super-enhancers in mouse and human genome. Nucleic Acids Res, 2016. 44(D1): p. D164-71.

46. Blinka, S., et al., Super-Enhancers at the Nanog Locus Differentially Regulate Neighboring Pluripotency-Associated Genes. Cell Rep, 2016. 17(1): p. 19-28.

47. Zabidi, M.A., et al., Enhancer-core-promoter specificity separates developmental and housekeeping gene regulation. Nature, 2015. 518(7540): p. 556-9.

48. Hounkpe, B.W., et al., HRT Atlas v1.0 database: redefining human and mouse housekeeping genes and candidate reference transcripts by mining massive RNA-seq datasets. Nucleic Acids Res, 2021. 49(D1): p. D947-D955.

49. Kim, J., et al., An extended transcriptional network for pluripotency of embryonic stem cells. Cell, 2008. 132(6): p. 1049-61.

50. Akagi, T., et al., ETS-related transcription factors ETV4 and ETV5 are involved in proliferation and induction of differentiation-associated genes in embryonic stem (ES) cells. J Biol Chem, 2015. 290(37): p. 22460-73.

51. Kinoshita, K., et al., GABPalpha regulates Oct-3/4 expression in mouse embryonic stem cells. Biochem Biophys Res Commun, 2007. 353(3): p. 686-91.

52. Zhang, X., et al., FOXO1 is an essential regulator of pluripotency in human embryonic stem cells. Nat Cell Biol, 2011. 13(9): p. 1092-9.

53. Wei, Z., et al., Klf4 interacts directly with Oct4 and Sox2 to promote reprogramming. Stem Cells, 2009. 27(12): p. 2969-78.

54. Bonev, B., et al., Multiscale 3D Genome Rewiring during Mouse Neural Development. Cell, 2017. 171(3): p. 557-572 e24.

55. Hsieh, T.S., et al., Resolving the 3D Landscape of Transcription-Linked Mammalian Chromatin Folding. Mol Cell, 2020. 78(3): p. 539-553 e8.

56. Kermekchiev, M., et al., Every enhancer works with every promoter for all the combinations tested: could new regulatory pathways evolve by enhancer shuffling? Gene Expr, 1991. 1(1): p. 71-81.

57. Gehrig, J., et al., Automated high-throughput mapping of promoter-enhancer interactions in zebrafish embryos. Nat Methods, 2009. 6(12): p. 911-6.

58. Tak, Y.E., et al., Augmenting and directing long-range CRISPR-mediated activation in human cells. Nat Methods, 2021. 18(9): p. 1075-1081.

59. El Khattabi, L., et al., A Pliable Mediator Acts as a Functional Rather Than an Architectural Bridge between Promoters and Enhancers. Cell, 2019. 178(5): p. 11451158 e20.

60. Fuqua, T., et al., Dense and pleiotropic regulatory information in a developmental enhancer. Nature, 2020. 587(7833): p. 235-239.

61. Kircher, M., et al., Saturation mutagenesis of twenty disease-associated regulatory elements at single base-pair resolution. Nat Commun, 2019. 10(1): p. 3583.

62. Pang, B. and M.P. Snyder, Systematic identification of silencers in human cells. Nat Genet, 2020. 52(3): p. 254-263.

63. Gisselbrecht, S.S., et al., Transcriptional Silencers in Drosophila Serve a Dual Role as Transcriptional Enhancers in Alternate Cellular Contexts. Mol Cell, 2020. 77(2): p. 324337 e8.

64. You, F.M., et al., BatchPrimer3: a high throughput web application for PCR and sequencing primer design. BMC Bioinformatics, 2008. 9: p. 253. 
bioRxiv preprint doi: https://doi.org/10.1101/2021.10.21.465269; this version posted October 22, 2021. The copyright holder for this preprint (which was not certified by peer review) is the author/funder, who has granted bioRxiv a license to display the preprint in perpetuity. It is made available under aCC-BY-NC-ND 4.0 International license.

871

872

873

874

875

876

877

878

879

880

881
65. Langmead, B. and S.L. Salzberg, Fast gapped-read alignment with Bowtie 2. Nat Methods, 2012. 9(4): p. 357-9.

66. Zorita, E., P. Cusco, and G.J. Filion, Starcode: sequence clustering based on all-pairs search. Bioinformatics, 2015. 31(12): p. 1913-9.

67. Diaferia, G.R., et al., Dissection of transcriptional and cis-regulatory control of differentiation in human pancreatic cancer. EMBO J, 2016. 35(6): p. 595-617.

68. Team, R.C., R: A Language and Environment for Statistical Computing. R Foundation for Statistical Computing, 2021. 2021.

69. Weirauch, M.T., et al., Determination and inference of eukaryotic transcription factor sequence specificity. Cell, 2014. 158(6): p. 1431-1443. 


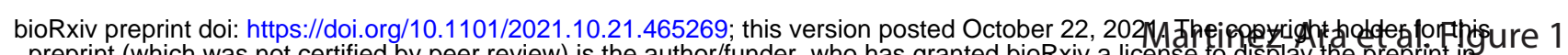
preprint (which was not certified by peer review) is the author/funder, who has granted bioRxiv a license

TADs

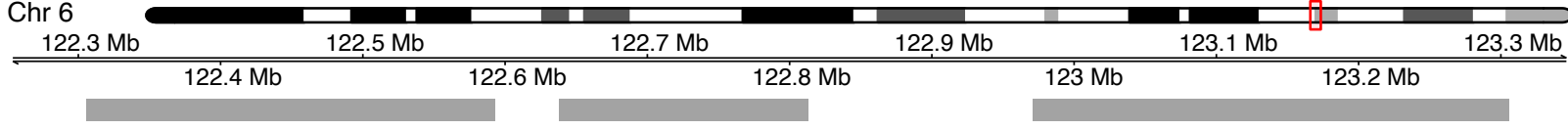

CCRES + Ps

Genes

B

Tfcp2l1 locus

$\begin{array}{ccccc}\text { Chr } 1118 \mathrm{Mb} & & 119 \mathrm{Mb} & 120 \mathrm{Mb} & \\ & & & 119.5 \mathrm{Mb} & 120.5 \mathrm{Mb}\end{array}$

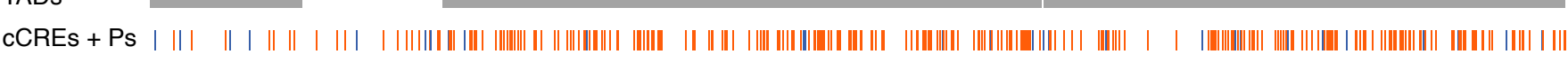
Nanog Genes

$$
\text { Tfcp2I1 }
$$

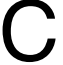

KLF2 locus

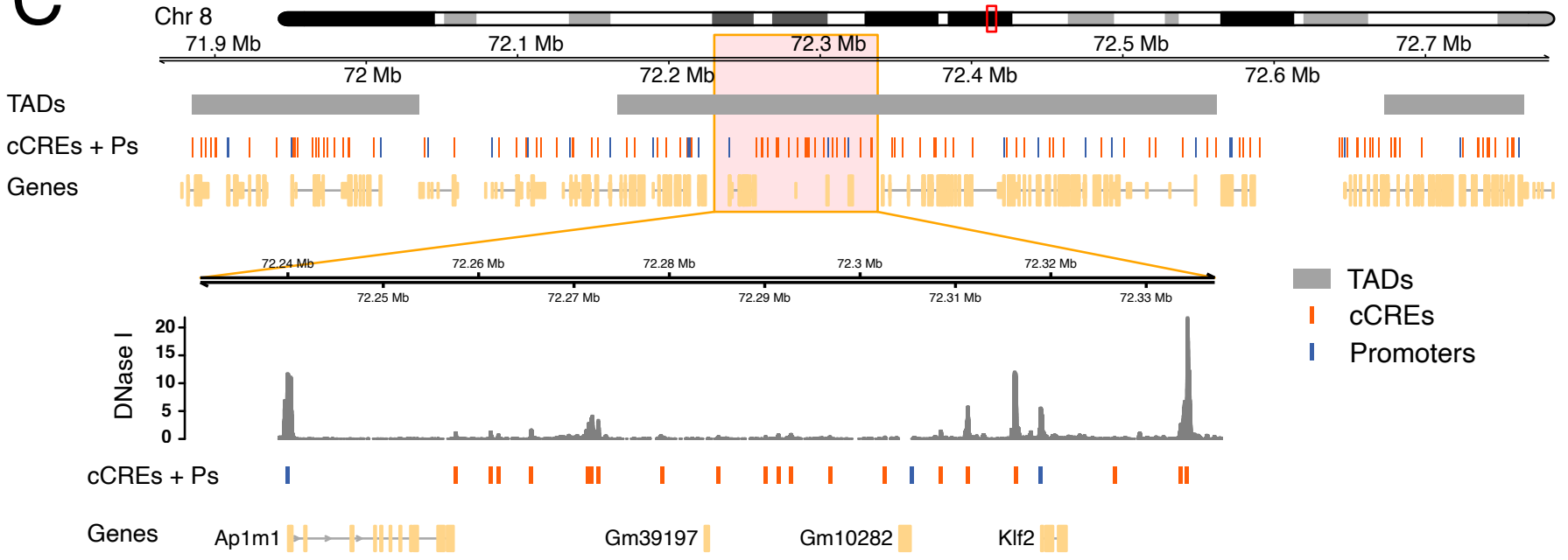

$\mathrm{D}$

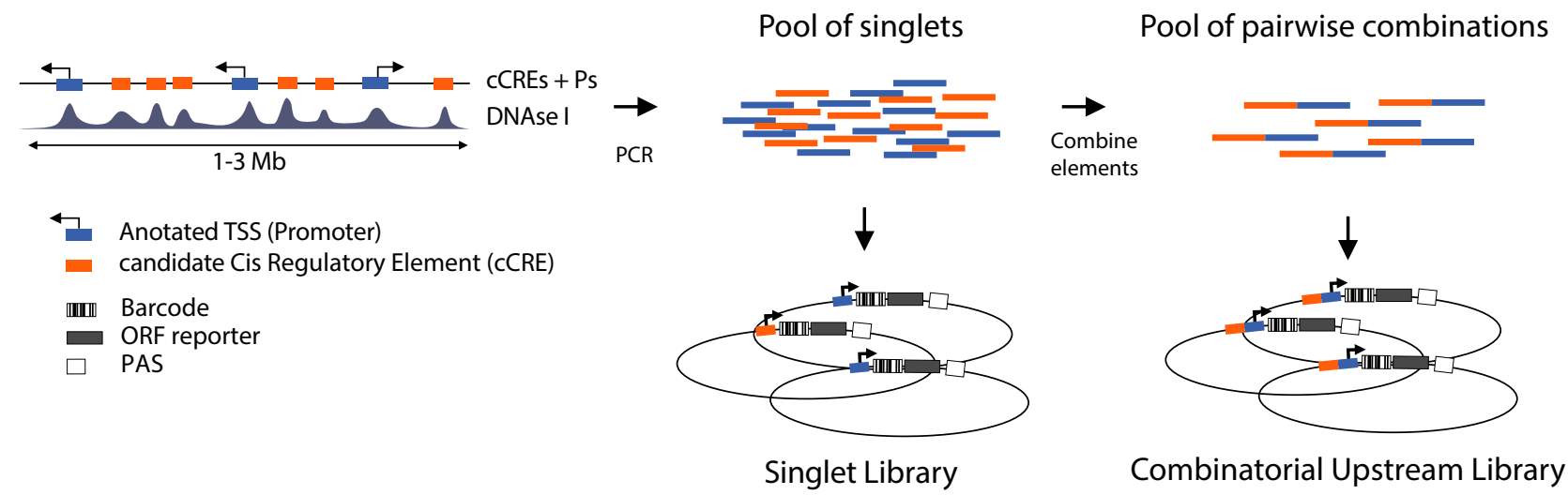

$E$

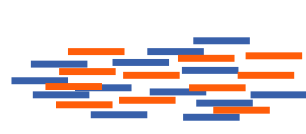

Pool of singlets

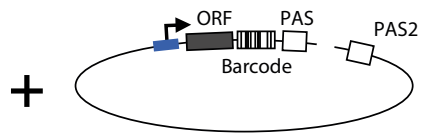

Downstream Vector

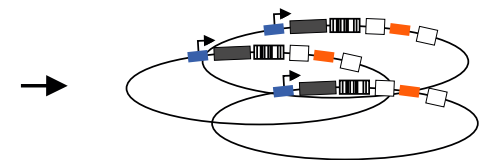

Combinatorial Downstream Library

Figure 1. Regulatory element selection and library construction. A-C) Representations of Nanog, Tfcp2l1, and KIf2 loci, respectively. In C) the zoom-in displays a DNAse I sensitivity track [38] where peaks overlap with cCREs. D) Cloning strategy for the Upstream assay. cCREs and promoters were amplified by PCR from genomic DNA and pooled. Fragments in this pool were then randomly ligated to generate duplets. Singlets and duplets were cloned into the same barcoded vector to generate two libraries per locus, a singlet library and a combinatorial library. E) Cloning strategy for the Downstream assay. The singlet pool from the KIf2 locus was cloned into ten vectors, each of them carrying a different promoter. The resulting ten sub-libraries were combined into one Downstream assay library. 


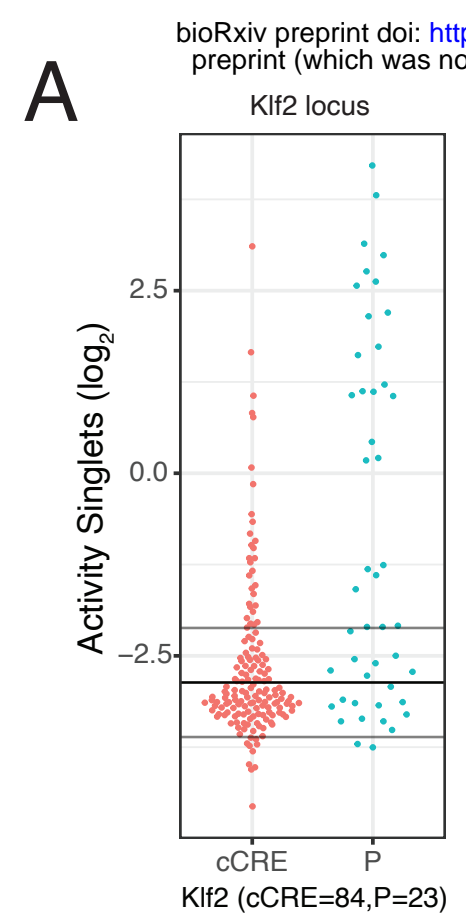

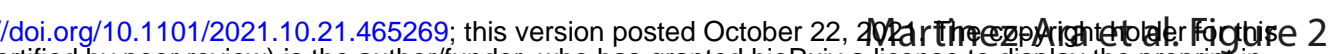
ertifipd by peer review) is the author/funder, who has granted bioRxiv a license to display the preprint in

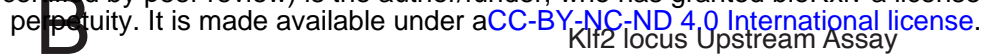

$\mathrm{C}$

$$
\text { 冚 }
$$

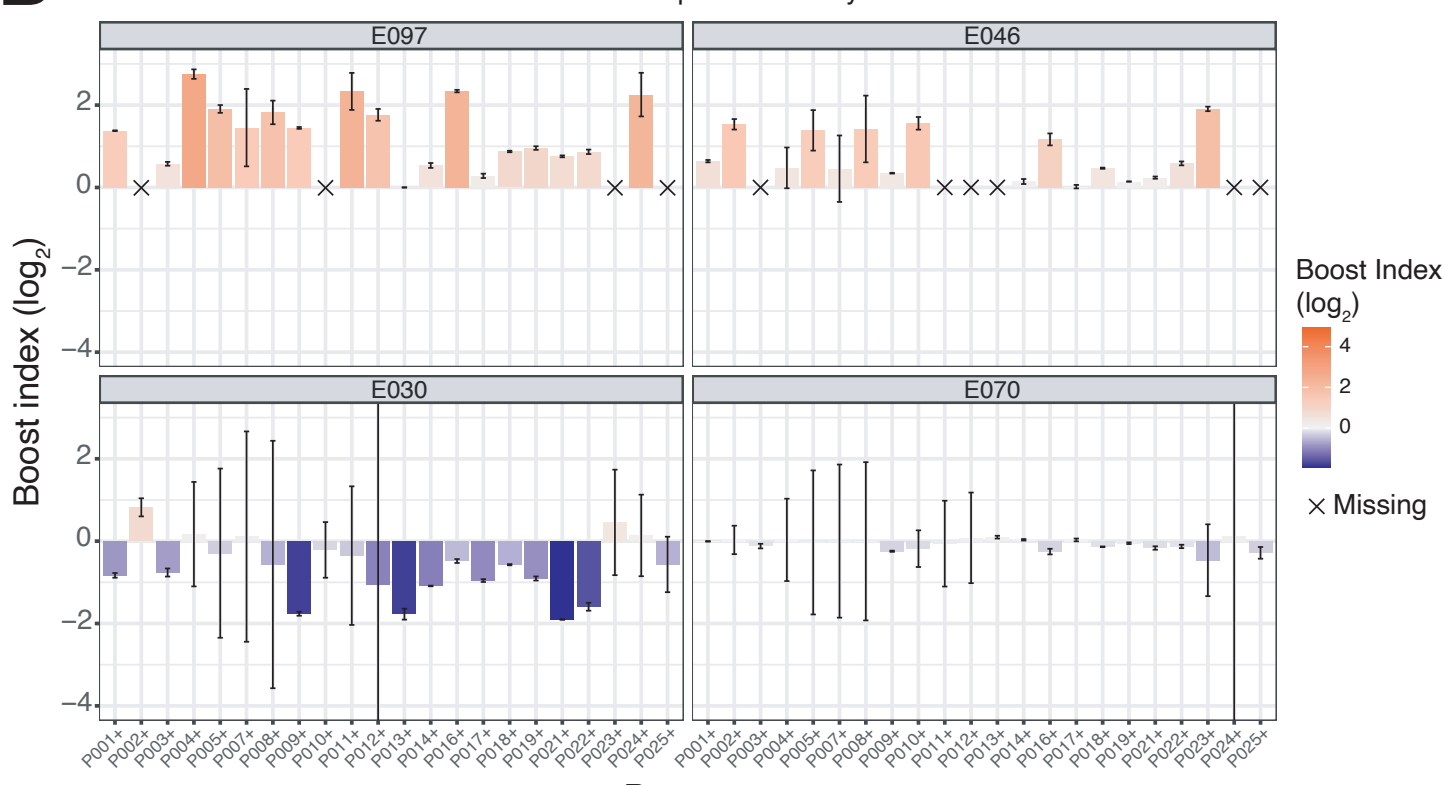

Promoter

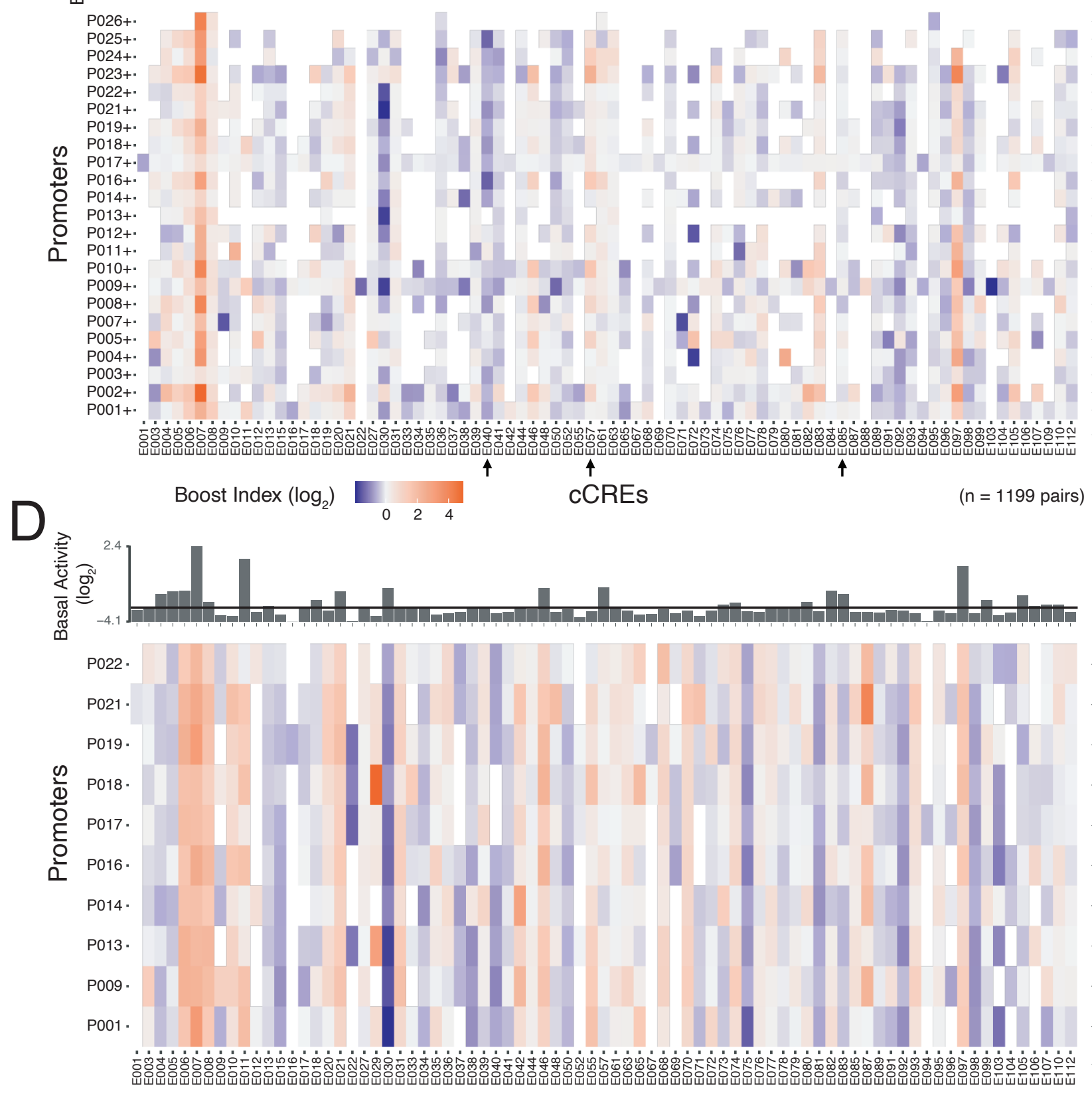

Klf2 locus Upstream assay

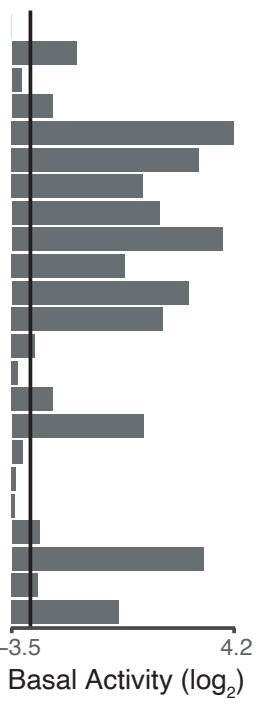

Klf2 locus Downstream assay

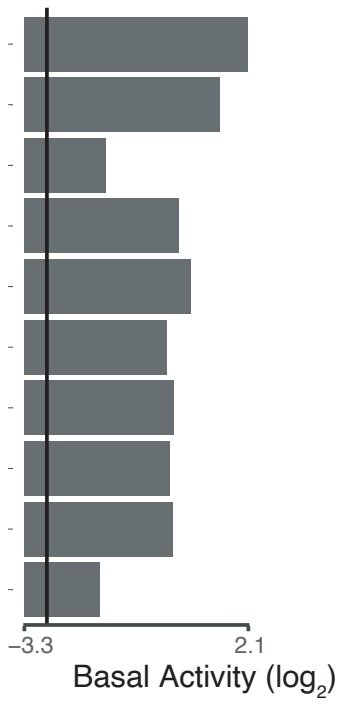


Figure 2. Singlet and combinatorial activities of cCREs and promoters from the Klf2 locus. A) Transcription activities of singlet cCREs and promoters. Each dot represents the mean activity of one singlet. Horizontal lines represent the average background activity of empty vectors (black line) plus or minus two standard deviations (grey lines). Elements with activities more than two standard deviations above the average background signal are defined as active. B) Examples of Upstream assay cCRE-P combinations for cCREs E097, E046, E030 and E070 of the KIf2 locus. Barplots represent the mean boost index of each combination, vertical lines represent the standard deviations. Crosses mark missing data. CD) Boost index matrices of cCRE-P combinations from the KIf2 locus according to Upstream (C) and Downstream (D) assays. White tiles indicate missing data. Barplots on the right and top of each panel show basal activities of each tested P or CCRE, respectively, with the black line indicating the background activity of the empty vector. All data are averages over 3 independent biological replicates. 


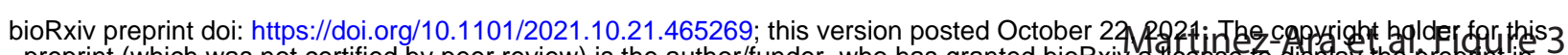

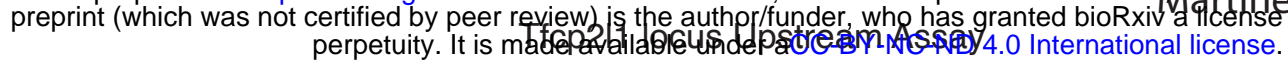

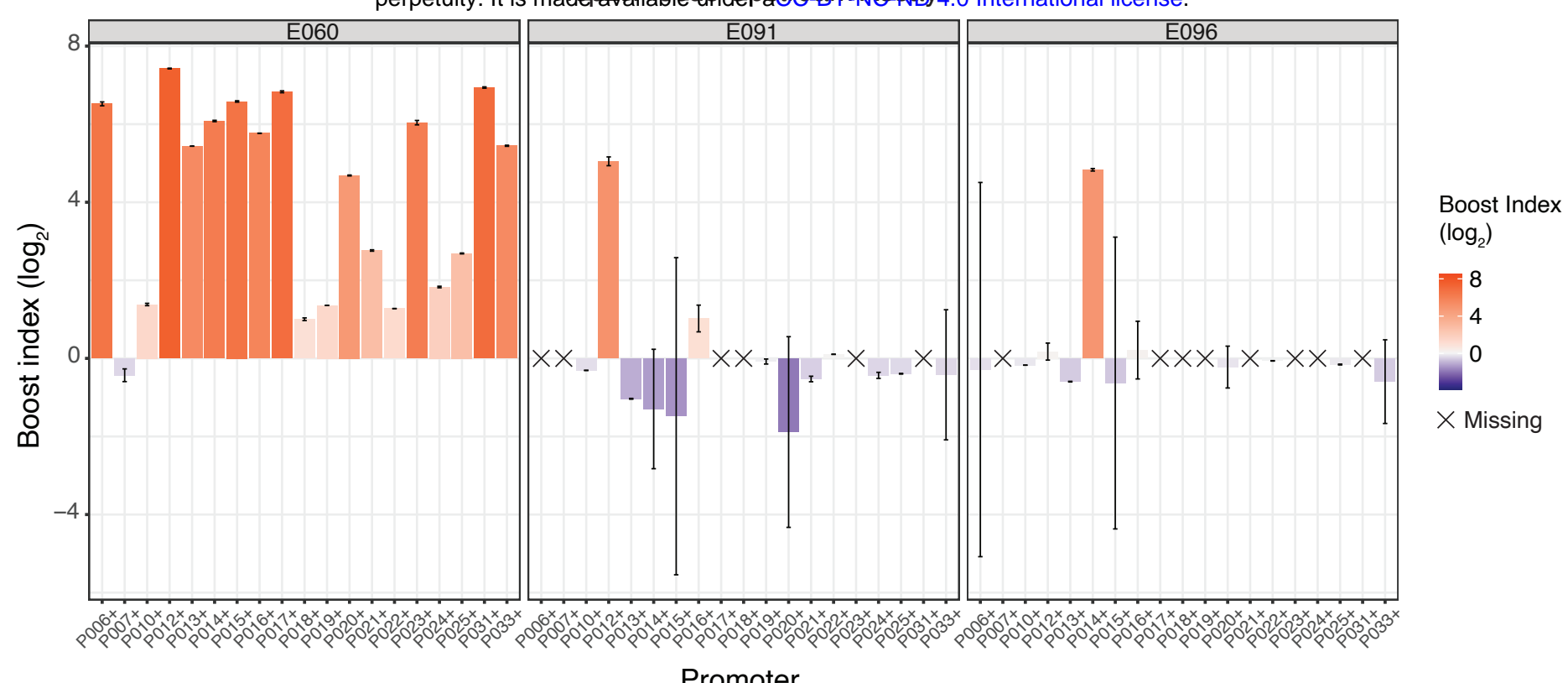

Figure 3. Examples of selective cCREs from the Tfcp2l1 locus. Boost indices obtained in the Upstream assay are shown for cCRE-P combinations of cCREs E060, E091 and E096 of the Tfcp2I1 locus. Barplots indicate the mean boost index of each combination, vertical lines indicate standard deviations. All data are averages over 3 independent biological replicates. 


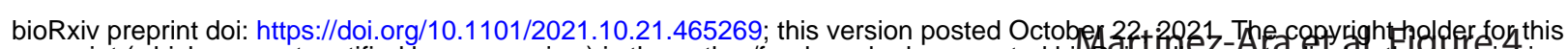

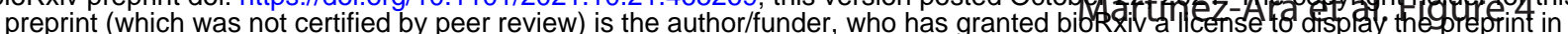

A

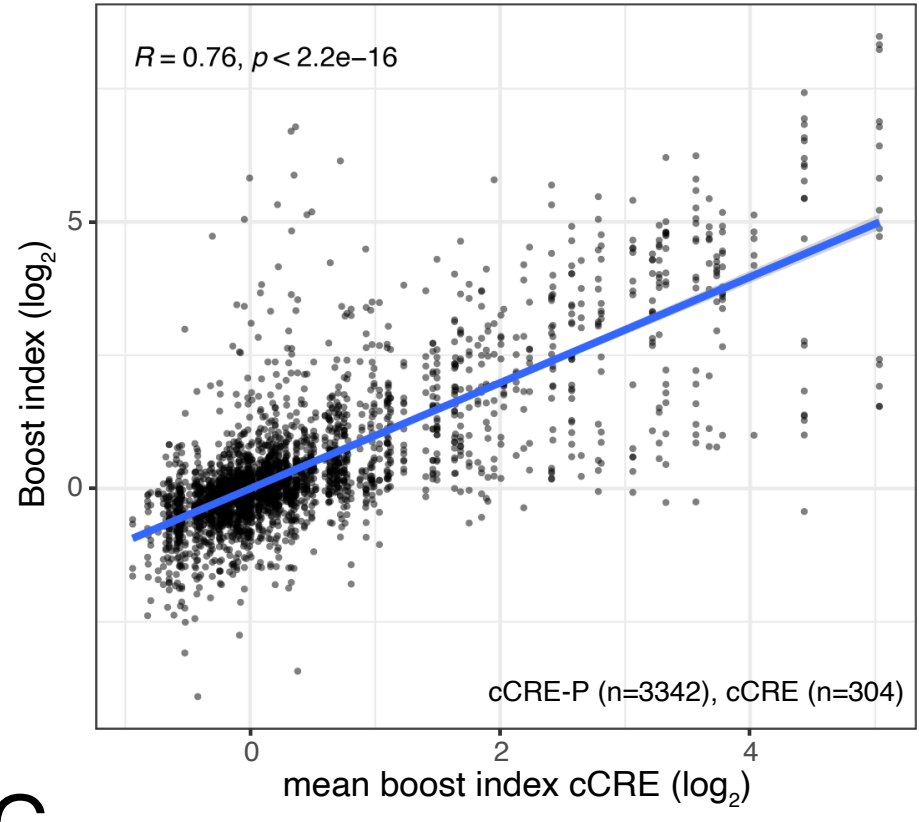

C

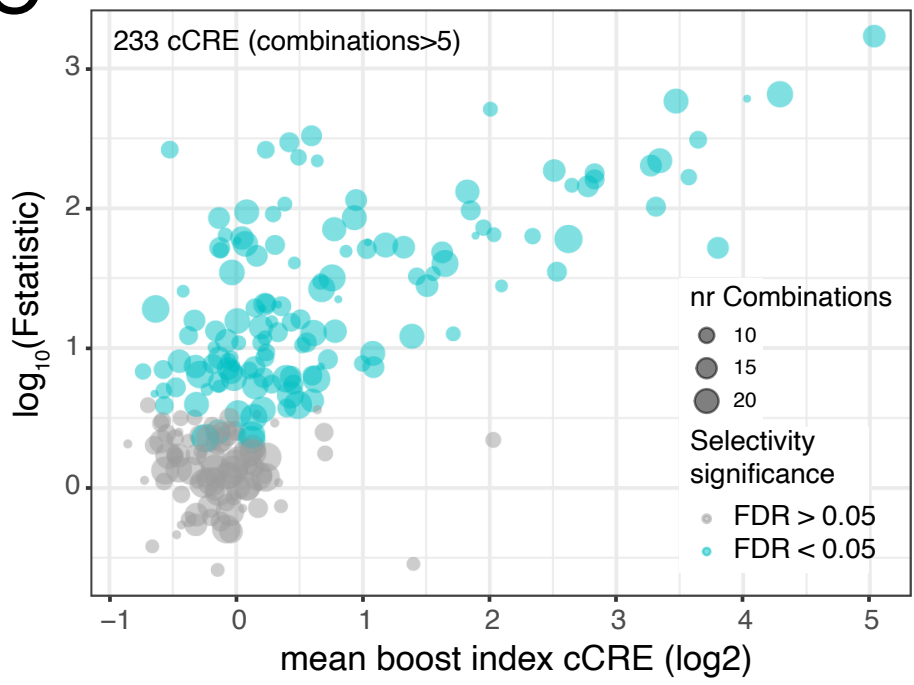

D

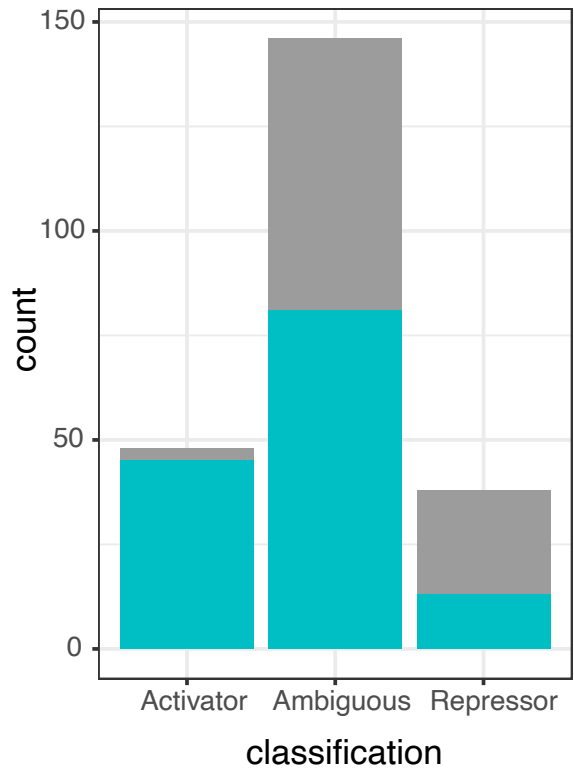

Selectity significance

FDR $>0.05$

FDR $<0.05$
B

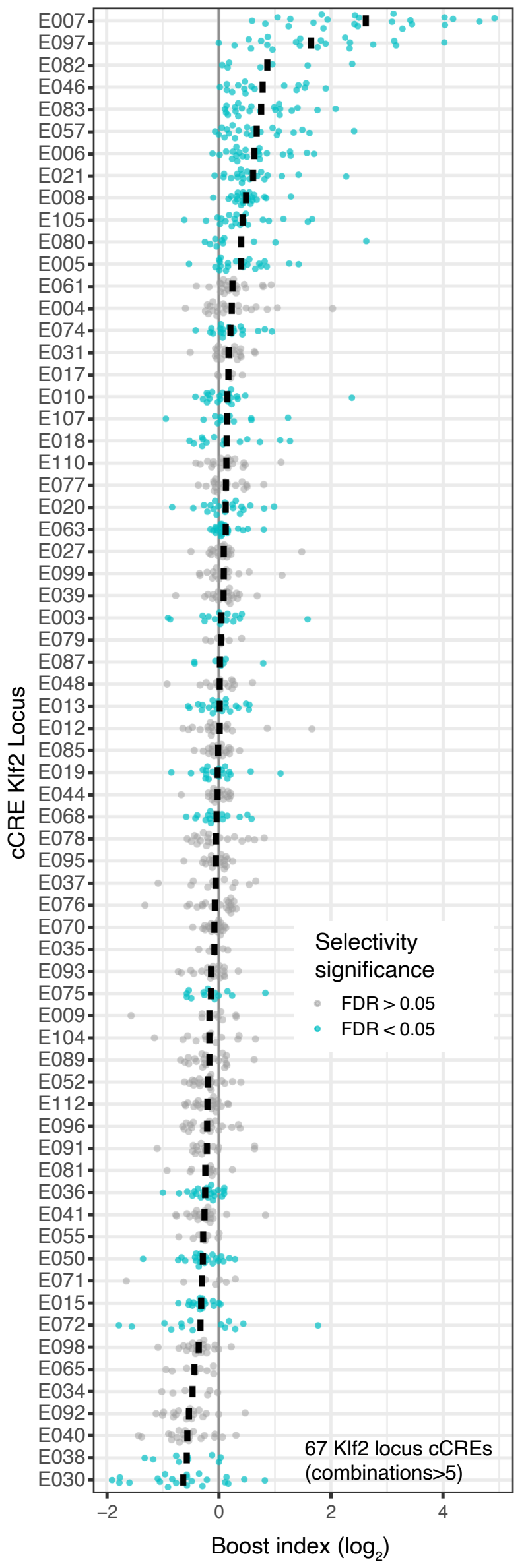


Figure 4. Promoter selectivity of cCREs. A) Plot showing the broad diversity of boost indices of many cCREs. Data are from Upstream assays of Klf2, Nanog and Tfcp2/1 loci combined. Vertical axis indicates boost indices of all tested CCRE-P pairs, which are horizontally ordered by the mean boost index of each CCRE. B) Boost index distributions for each cCRE from the KIf2 locus (Upstream assay). Each dot represents one cCRE-P combination; black bar represents the mean. Turquoise colouring marks cCREs that have a larger variance of their boost indices than may be expected based on experimental noise, according to the Welch F-test after multiple hypothesis correction (5\% FDR cutoff). C) Summary of Welch Ftest selectivity analysis results for all cCREs from the three loci with more than 5 cCRE-P combinations. Each dot represents one $C C R E$; the size of the dots indicates the number of cCRE-P pairs. Significantly selective cCREs (5\% FDR cutoff) are highlighted in turquoise. D) Proportion of significantly selective (turquoise) CCRE in the three categories as shown in Figure S3A. All data are averages over 3 independent biological replicates. 
bioRxiv preprint doi: https://doi.org/10.1101/2021.10.21.465269; this version posted October 22, 2021. The copyright holder for this

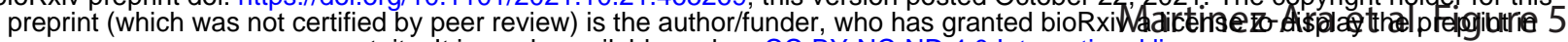
perpetuity. It is made available under aCC-BY-NC-ND 4.0 International license.

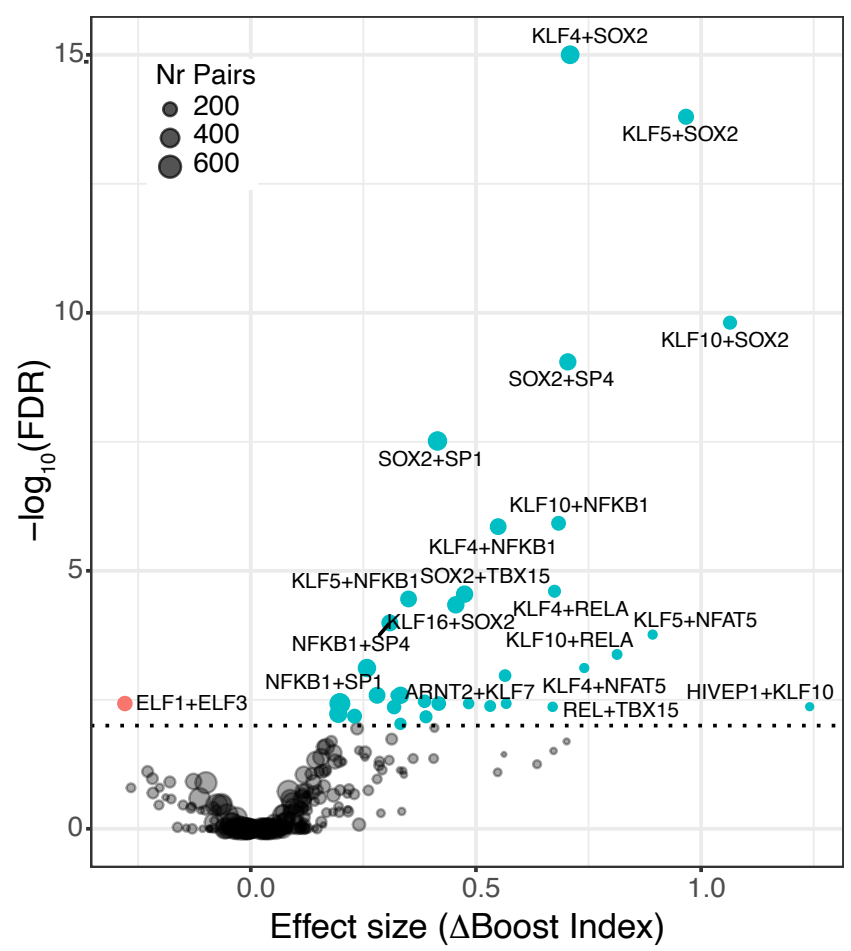

TF1 TF2

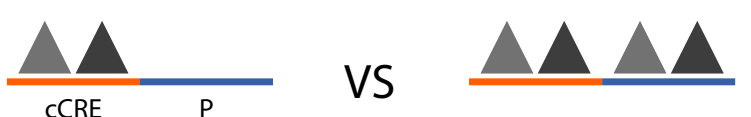

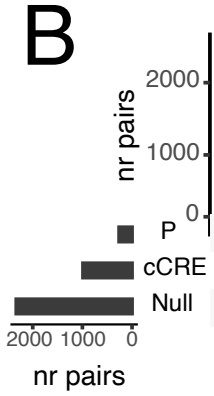

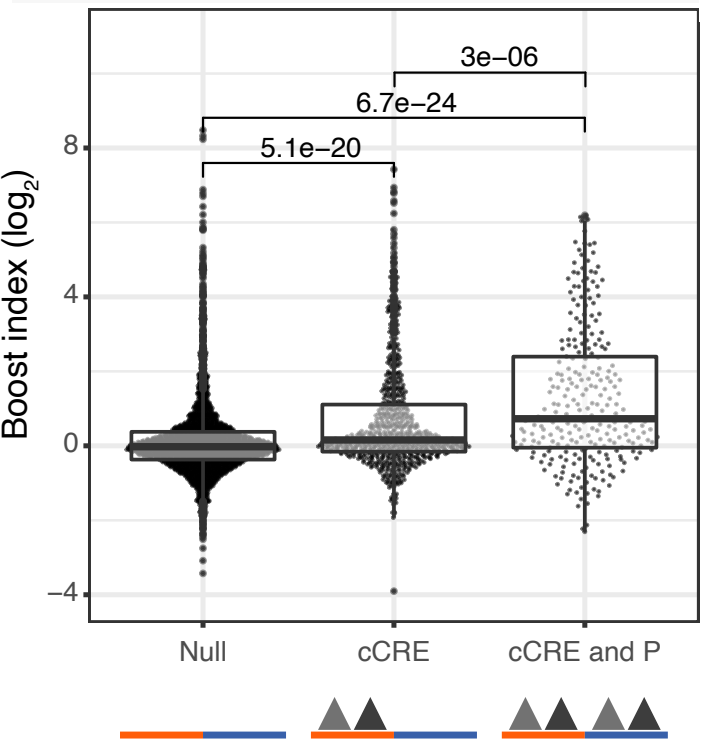

KLF4 + Sox2 motif presence

Figure 5. Association of TF motif Duos with higher boost indices. A) Results of TF survey for self-compatible TF motif Duos. TF motif duos associated with higher or lower boost indices at a 1\% FDR cutoff are highlighted. B) Association of Sox2+KIf4 motifs at both cCRE and P with higher boost indices. cCRE-P combinations are split into 3 groups according to presence or absence of Sox2+Klf4 motifs both at the cCRE and the promoter, or only the cCRE. Numbers at the top of horizontal brackets are the p-values obtained from comparing the different groups boost index distributions using a Wilcoxon rank-sum test. Boxplots represent median and interquartile ranges. Barplots at the top represent the number of combinations in each group. 


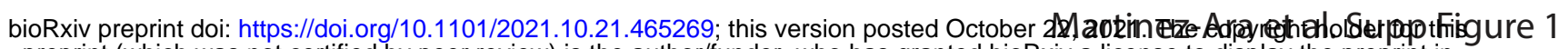
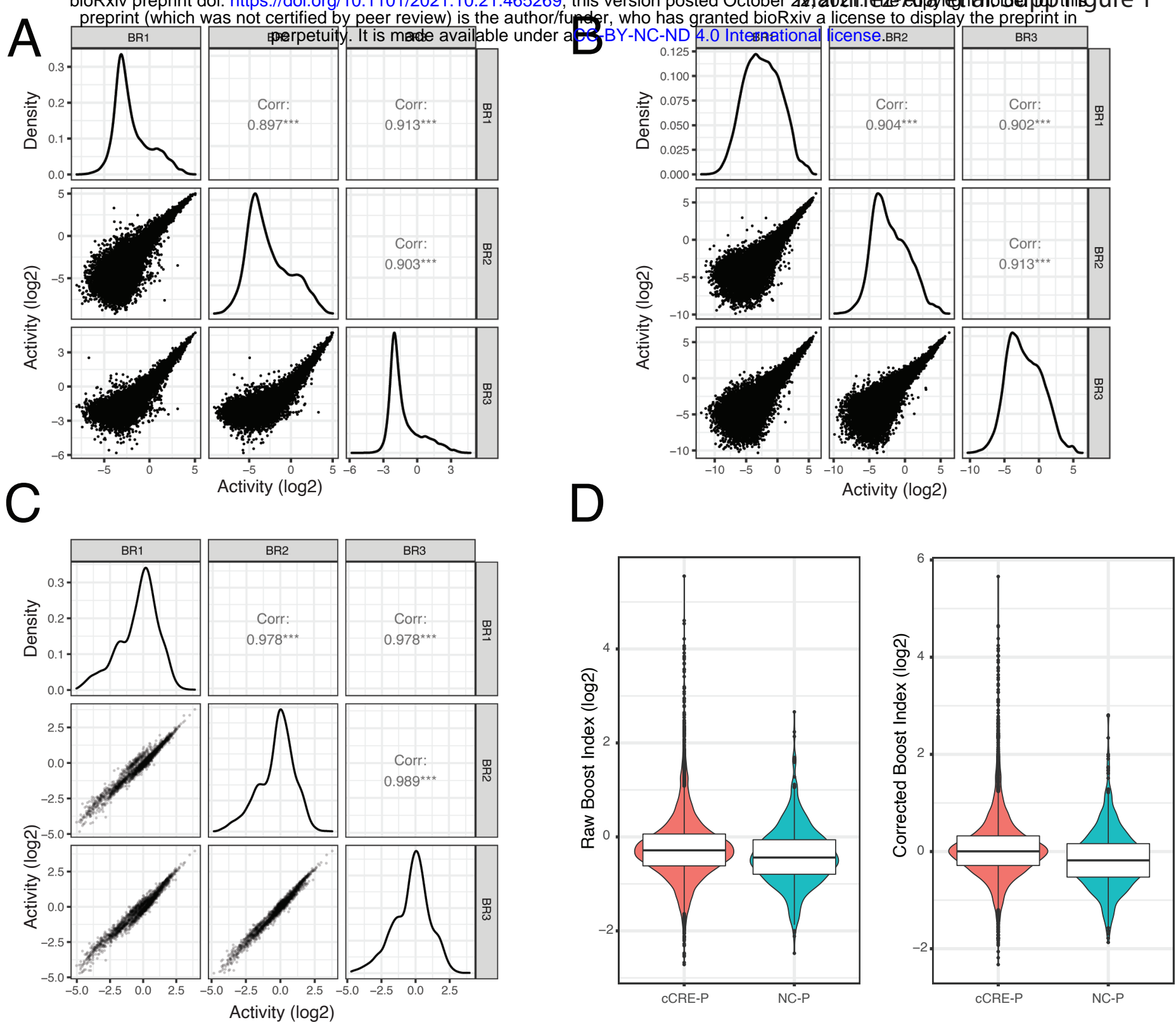

Figure S1. Reproducibility of data and boost index calculation. (A-C) Correlograms of the three biological replicates of each library pool. Lower left panels show pairwise scatterplots of the activities of all cCRE-P pairs per replicate. Middle panels show the density of data distribution in each replicate and upper right panels show the Pearson correlation coefficients. A) Klf2 and Nanog Upstream libraries. B) Tfcp2l1 Upstream library. C) Klf2 Downstream libraries. D) Upstream assay boost index distributions for cCRE-P and negative controls - promoter (NC-P) combinations. Left panel: raw boost indices; right panel: boost indices after correction for negative bias (see Methods). 


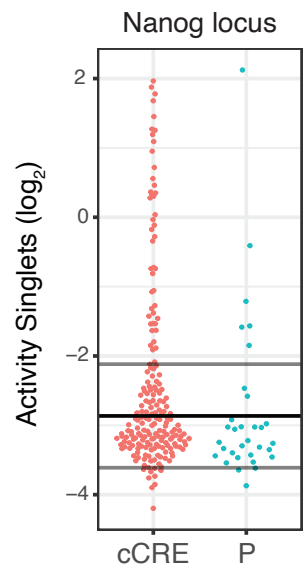

$\operatorname{Nanog}(E=104, P=18)$
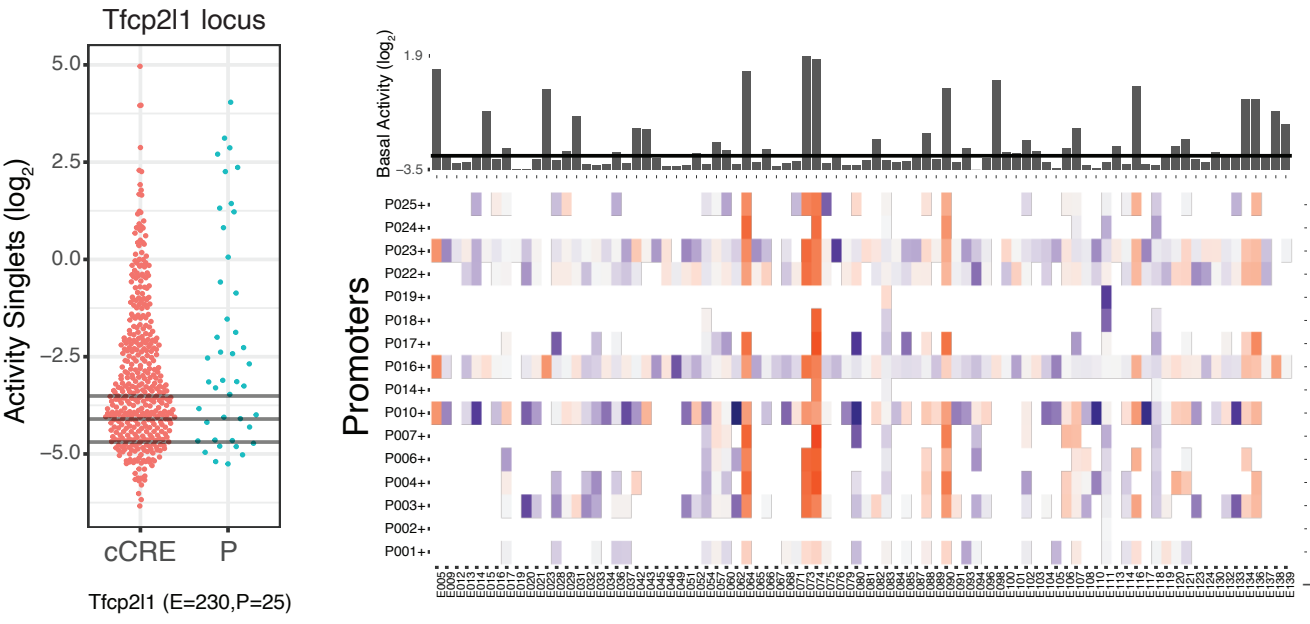

Nanog locus Upstream assay
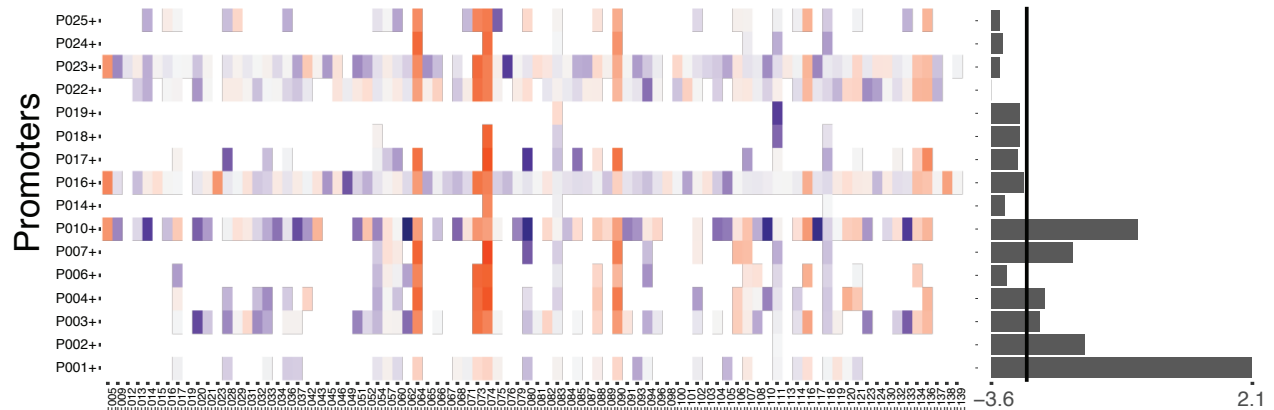

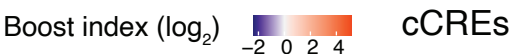

( $\mathrm{n}=501$ pairs $)$

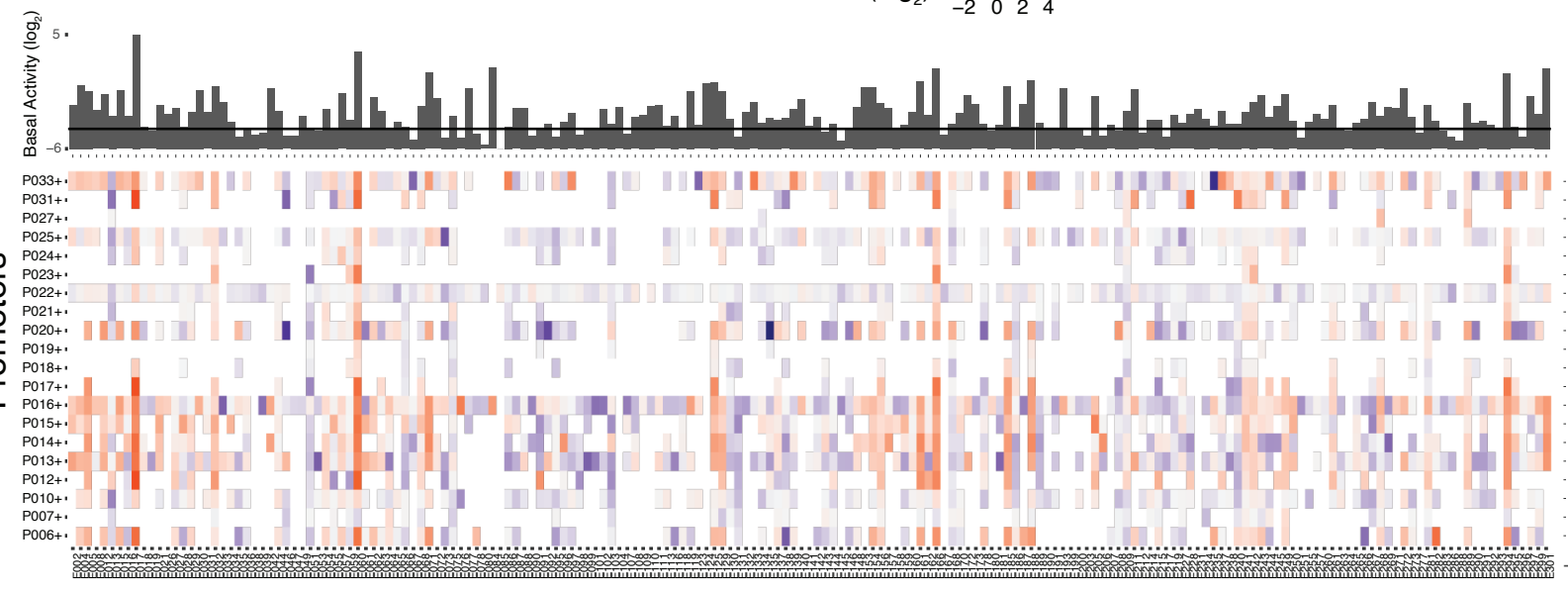

Boost Index $\left(\log _{2}\right)$
CCRES $(\mathrm{n}=1625$ pairs $)$
Tfcp2l1 locus Upstream assay

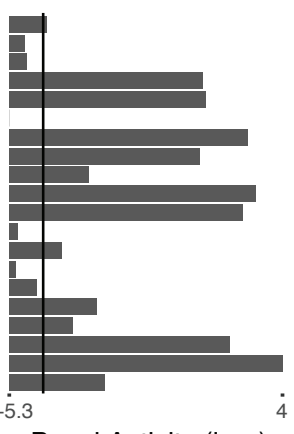

Basal Activity $\left(\log _{2}\right)$

$\mathrm{D}$

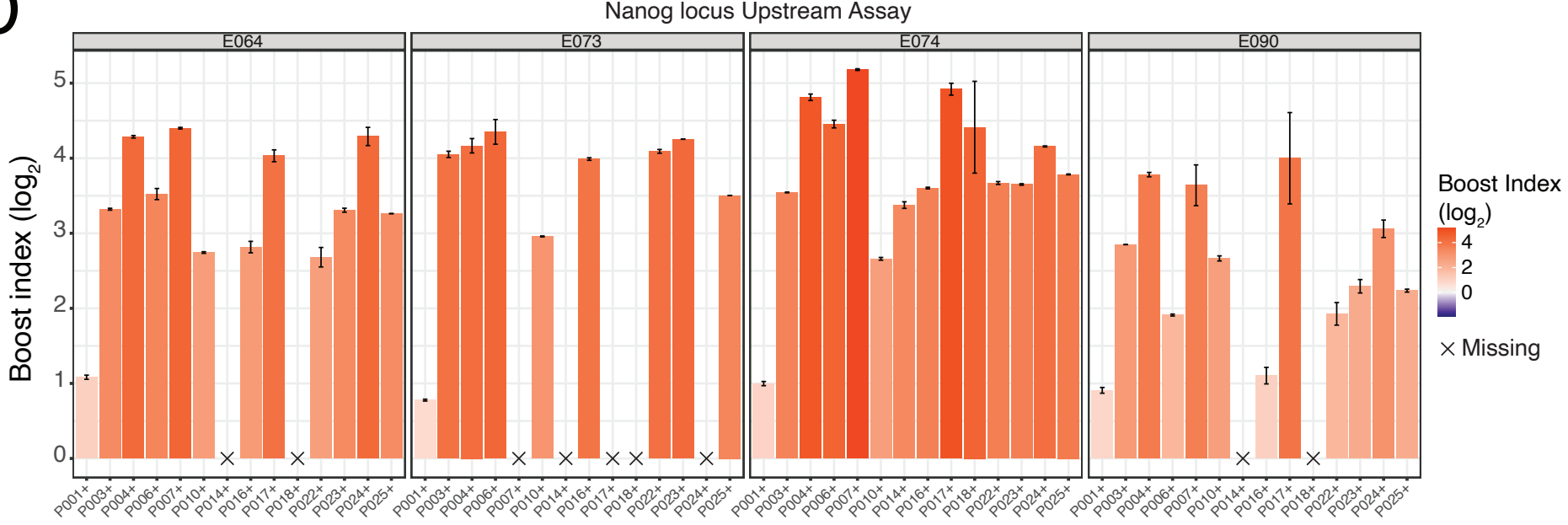

Promoter

Figure S2. Element activities and boost indices obtained with Nanog and Tfcp2I1 Upstream libraries. A) Transcriptional activities of cCREs and promoters. Each dot represents the mean activity of one singlet. Horizontal lines represent the average background activity of empty vectors (black line) plus or minus two standard deviations (grey lines). Elements with activities more than two standard deviations above the average background signal are defined as active. B-C) Boost index matrices for CCRE-P pairs from Nanog and Tfcp2l1 loci (both Upstream assays). White tiles indicate missing data. Barplots on the right and top of each panel show basal activities of each tested $\mathrm{P}$ or CCRE, respectively, with the black line indicating the background activity of the empty vector. D) Examples of cCRE-P combinations for cCREs E064, E073, E074 and E090 of the Nanog locus. Barplots represent the mean boost index of each combination, vertical lines represent the standard deviation of each boost index. All data are averages over 3 independent biological replicates. 
bioRxiv preprint doi: https://doi.org/10.1101/2021.10.21.465269; this version posted October 22 2021. The cgpyright hqlder for this.

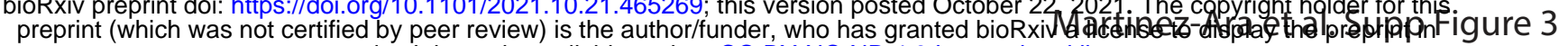
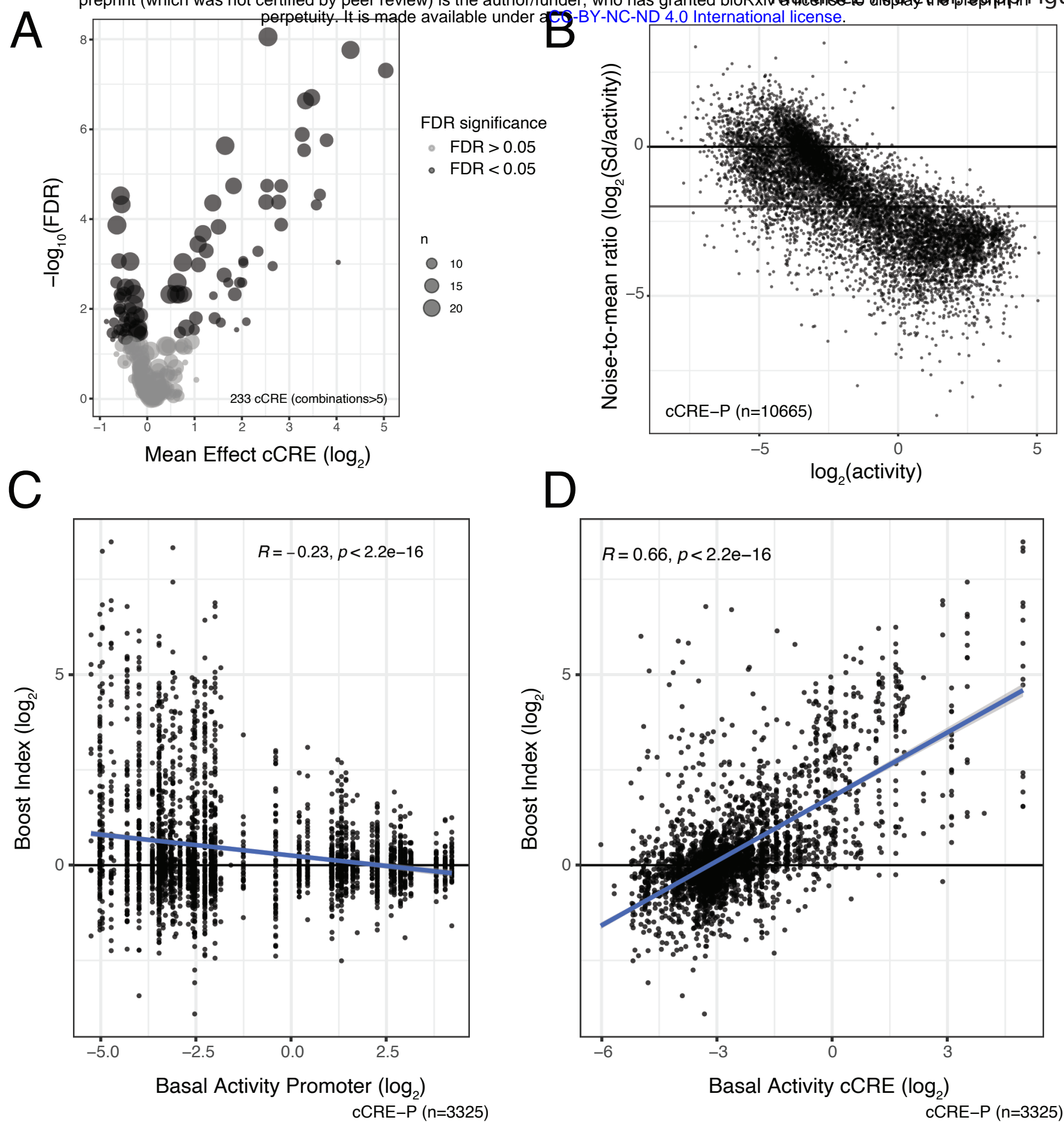

cCRE-P $(n=3325)$

Figure S3. cCRE functional classification and activity influence on Boost indices. A) Volcano plot of cCREs associated with activation or repression across promoters. A Wilcoxon test is performed per cCRE comparing the boost indices of all the cCRE-P combinations of that CCRE against the rest of CCRE-P combinations. A minimum of 6 combinations is required per cCRE. P-values are corrected for multiple hypothesis testing using the Benjamini-Hochberg method (FDR). B) Relationship between noise-to-mean ratio (Standard Deviation/mean Activity) and mean activity of cCRE-Ps. Horizontal lines represent noise-to-mean ratios of 1 and of 4 in log2 scale. C) Relationship between boost indices and basal (singlet) P activity. Each column of dots shows the data of cCRE-P pairs for one P. Data are from Upstream assays of all three loci combined. D) Relationship between boost indices and basal (singlet) cCRE activity. All data are averages over 3 independent biological replicates. 

perpetuity. It is made available under -BY-NC-ND 4.0 International license.

Upstream Assay Orientation Independence

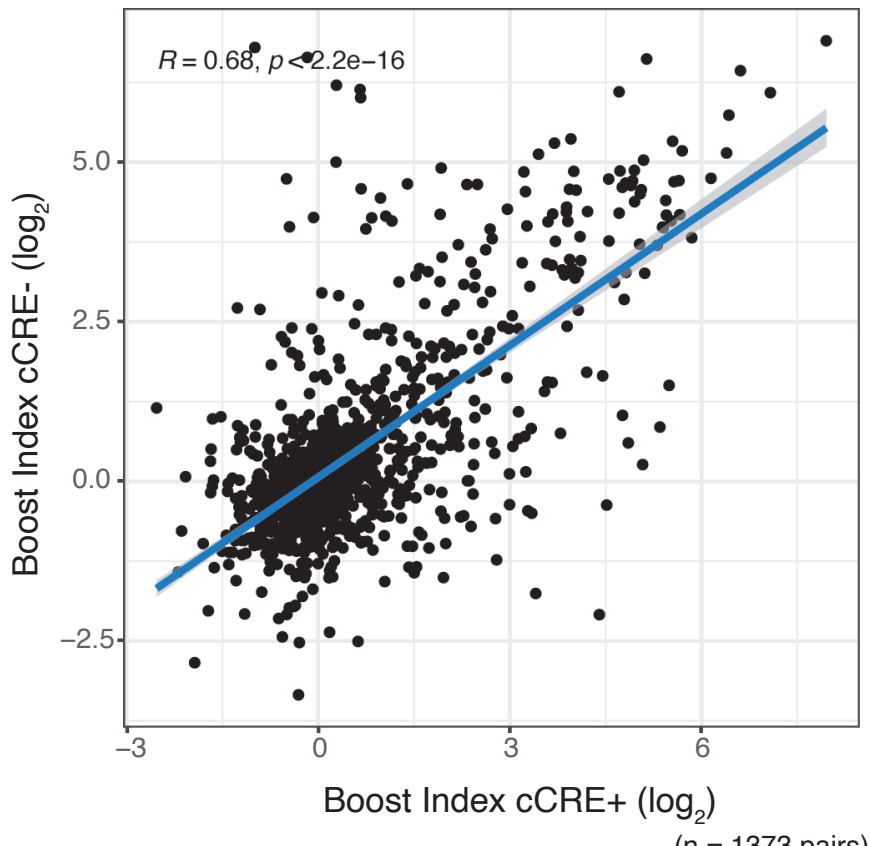

Downstream assay Orientation Independence

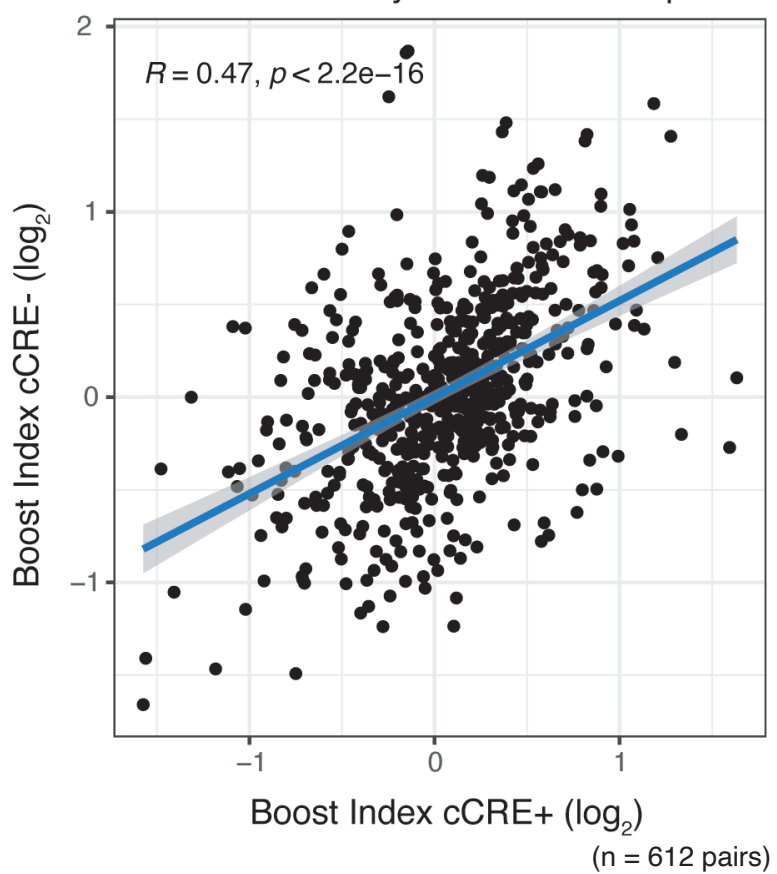

Position Independence

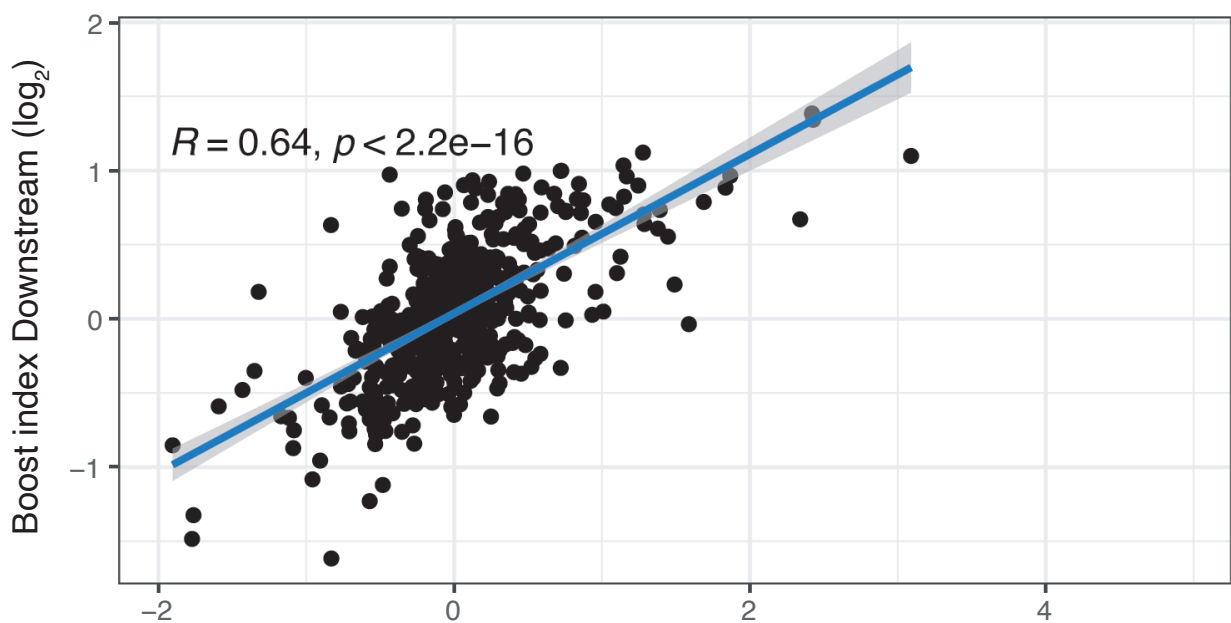

Boost index Upstream $\left(\log _{2}\right)$

( $\mathrm{n}=1199$ pairs)

Figure S4. Orientation and position independence of cCREs. (A-B) Correlation between boost indices of both cCRE orientations of the same CCRE-P combination, in the (A) Upstream assay and (B) Downstream assay. Data are from the KIf2 locus libraries. Note that "+" and "-" orientations are arbitrary labels, because cCREs do not have an intrinsic orientation. (C) Correlation between boost indices of cCRE-P combinations shared between the Upstream and Downstream assays of the Klf2 locus. In all panels $R$ is the Pearson correlation coefficient. All data are averages over 3 independent biological replicates. In C Boost indices are averaged over cCRE orientations. 
bioRxiv preprint doi: https://doi.org/10.1101/2021.10.21.465269; this version posted October 22, 2021.. The copyright holder for this.

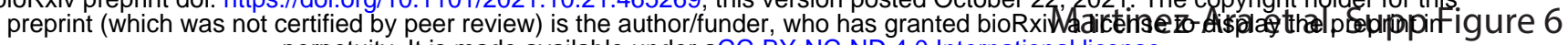

A perpetuity. It is made available under aCC-BY-NC-ND 4.0 International license.

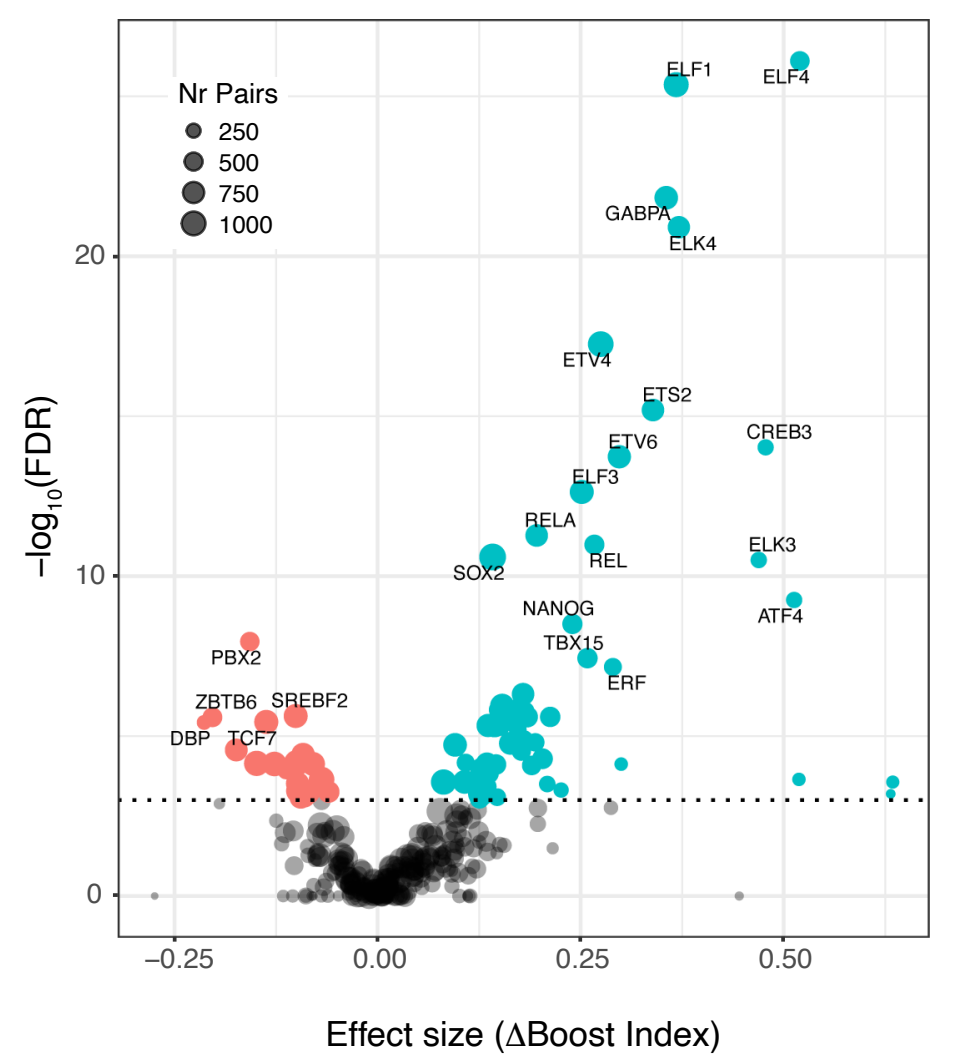

CCRE $\quad \mathrm{P}$ VS
B

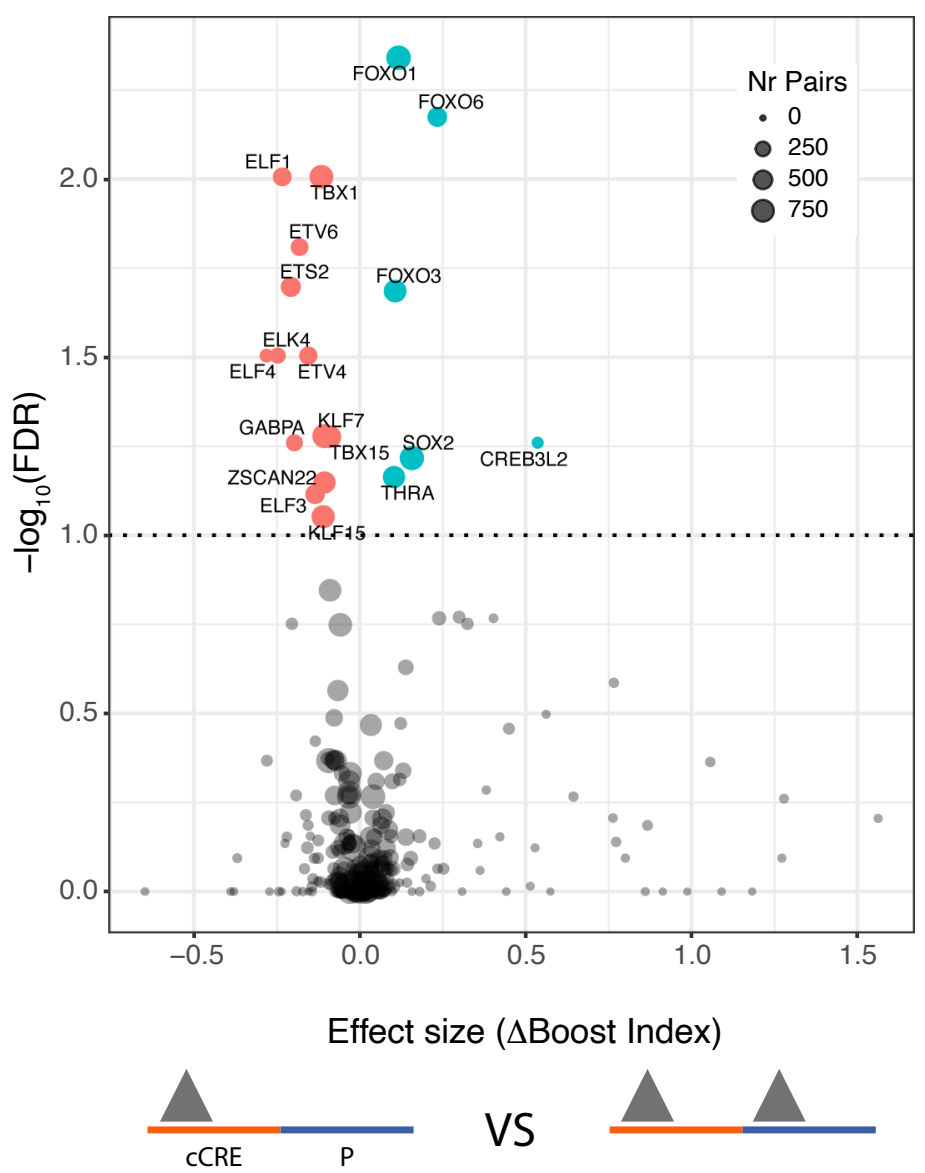

Figure S6. Identification of single TF motifs that correlate with boost indices. (A) TF motifs in cCREs associated (at 1\% FDR cutoff) with activation (turquoise) or repression (red). (B) Motifs of putative self-compatible TFs, i.e. motifs that predict increased or reduced boosting indices when present both at the cCRE and P, compared to being present only at the CCRE. TF motifs associated with higher or lower boost indices at a $1 \%$ FDR cutoff are highlighted. We note that TF motifs with multiple hits from the same family, such as for ELK, FOXO and ELF factors, may in fact be due to the activity of one TF motif of that family [69]. 